Publ. Mat. 47 (2003), 311-358

\title{
DISCRETIZATION AND ANTI-DISCRETIZATION OF REARRANGEMENT-INVARIANT NORMS
}

\author{
Amiran Gogatishvili And Luboš PiCK
}

Abstract

We develop a new method of discretization and anti-discretization of weighted inequalities which we apply to norms in classical Lorentz spaces and to spaces endowed with the so-called Hilbert norm. Main applications of our results include new integral conditions characterizing embeddings $\Gamma^{p}(v) \hookrightarrow \Gamma^{q}(w)$ and $\Gamma^{p}(v) \hookrightarrow \Lambda^{q}(w)$ and an integral characterization of the associate space to $\Gamma^{p}(v)$, where $p, q \in(0, \infty), v, w$ are weights on $[0, \infty)$ and

$$
\begin{aligned}
& \|f\|_{\Lambda^{p}(v)}=\left(\int_{0}^{\infty} f^{*}(t)^{p} v(t) d t\right)^{1 / p}, \\
& \|f\|_{\Gamma^{p}(v)}=\left(\int_{0}^{\infty} f^{* *}(t)^{p} v(t) d t\right)^{1 / p} .
\end{aligned}
$$

\section{Introduction}

Let $(\mathcal{R}, \mu)$ be a totally $\sigma$-finite measure space with a non-atomic measure $\mu$, and let $\mathcal{M}(\mathcal{R}, \mu)$ be the set of all extended complex-valued $\mu$-measurable functions on $\mathcal{R}$. We shall throughout assume that $\mu(\mathcal{R})=\infty$. For $f \in \mathcal{M}(\mathcal{R}, \mu)$, let $f_{*}(t)=\mu(\{x \in \mathcal{R} ;|f(x)|>t\}), t \in(0, \infty)$, be the distribution function of $f$. The non-increasing rearrangement of $f$ is defined by

$$
f^{*}(t)=\inf \left\{s>0 ; f_{*}(s) \leq t\right\}, \quad t \in[0, \infty) .
$$

2000 Mathematics Subject Classification. 26D10, 46E20.

Key words. Discretizing sequence, Hilbert norm, classical Lorentz space, duality theorems.

This research was partly supported by the grant no. 201/01/0333 of the Grant Agency of the Czech Republic and by the Leverhulme Trust Grant no. F/00407/E. The research of the second author was partly supported by the grant no. MSM 113200007 of the Czech Ministry of Education. 
We further set

$$
f^{* *}(t)=\frac{1}{t} \int_{0}^{t} f^{*}(s) d s, \quad t \in[0, \infty) .
$$

When $v$ is a non-negative measurable function on $[0, \infty)$, we say that $v$ is a weight.

Definition 1.1. Let $p \in(0, \infty)$ and let $v$ be a weight. Then the classical Lorentz space $\Lambda^{p}(v)$ is defined as

$$
\Lambda^{p}(v)=\left\{f \in \mathcal{M}(\mathcal{R}, \mu) ;\|f\|_{\Lambda^{p}(v)}:=\left(\int_{0}^{\infty} f^{*}(t)^{p} v(t) d t\right)^{1 / p}<\infty\right\} .
$$

Let us recall that $\|\cdot\|_{\Lambda^{p}(v)}$ is not always a norm (consider, for example, the cases when $p \in(0,1)$ ), and, indeed, not even a quasinorm (cf. [8, Corollary 2.2]).

The spaces $\Lambda^{p}(v)$ were introduced by Lorentz in 1951 in [21]. For appropriate values of $p$ and for appropriate weight $v$ this space is a rearrangement-invariant Banach function space (for definitions and detailed study of rearrangement-invariant Banach function spaces see e.g. [3]).

Another type of a function space is obtained by replacing in the definition on $\|\cdot\|_{\Lambda^{p}(v)}$ the $f^{*}$ with $f^{* *}$. This space, denoted by $\Gamma^{p}(v)$, is defined as

$$
\Gamma^{p}(v)=\left\{f \in \mathcal{M}(\mathcal{R}, \mu) ;\|f\|_{\Gamma^{p}(v)}:=\left(\int_{0}^{\infty} f^{* *}(t)^{p} v(t) d t\right)^{1 / p}<\infty\right\} .
$$

The spaces $\Gamma^{p}(v)$ proved to be quite useful in many branches of functional analysis, for example in interpolation theory. They are particularly popular since 1990 when Sawyer [26] used them in order to characterize certain duality properties of spaces $\Lambda^{p}(v)$, but they can be traced in earlier works of Calderón, Hunt, O'Neil and others.

Naturally, we have seen an extensive research of classical Lorentz spaces during the 1990's. Above all, the authors concentrated on seeking manageable and easily-verifiable necessary and sufficient conditions for embedding theorems involving both $\Lambda$ and $\Gamma$ types of spaces. Such results have an intimate connection to other challenging problems. Let us name some of them:

Is a given classical Lorentz space (either of type $\Lambda$ or of type $\Gamma$ ) a Banach space?

Is the Hardy-Littlewood maximal operator bounded on a given classical Lorentz spaces?

What is the associate space of $\Gamma^{p}(v)$ ? 
All these questions could be answered if only we knew necessary and sufficient conditions on embeddings between $\Lambda$ and $\Gamma$ types of spaces. The research brought plenty of deep results, cf. e.g. [1], [26], [8], [9], $[\mathbf{1 0}],[\mathbf{1 6}],[\mathbf{3 0}],[\mathbf{3 1}],[\mathbf{3 2}],[\mathbf{2 8}]$. A summary of the results on embeddings of classical Lorentz spaces known by the end of 1990's, as well as some more references, can be found in [7]. For some cases of the parameters, the characterization of the corresponding embedding is still not known. In some other cases necessary and sufficient conditions have been established, but formulated in a way which is not entirely satisfactory, as they might be quite difficult to verify. Typical examples of such conditions are those expressed in terms of the Halperin level function [7, Section 7] or in terms of discretizing sequences [16].

In [16], a new approach based on discretization techniques of [25] and [15] was applied to classical Lorentz spaces in order to obtain necessary and sufficient conditions on parameters $p, q \in(0, \infty)$ and weights $v, w$ such that the embedding $\Gamma^{p}(v) \hookrightarrow \Gamma^{q}(w)$ or the embedding $\Gamma^{p}(v) \hookrightarrow \Lambda^{1}(w)$ hold. The former embedding is useful in interpolation theory while a standard argument applied to the latter provides a characterization of the associate space to $\Gamma^{q}(w)$. The results of $[\mathbf{1 6}]$ meant a considerable step ahead. However, to verify the conditions formulated through the discretizing sequences is almost impossible. After [16] was published many authors tried to obtain more manageable conditions (expressed, if possible, in an "integral form" - such conditions have been successfully used for example to characterize weighted Hardy inequalities). As far as we know, no such results have been found so far. In this paper we develop a new method that leads to such results.

Our approach is based on discretization and (more importantly) antidiscretization methods combined with the blocking technique from [17]. Let us outline our approach and the structure of the paper. We start with general discretization and anti-discretization formulae for weighted integral norms. This is done in Section 2. Next, in Section 3, we present a discretization of the so-called Hilbert norm. Let us recall that, in a very general form, the Hilbert norm of a function $f$ is the quantity

$$
\left(\int_{0}^{\infty} \varphi(x)\left(\int_{0}^{\infty} \frac{|f(y)|}{u(x)+u(y)} d y\right)^{q} \varphi(x) d x\right)^{\frac{1}{q}}
$$

when $q \in(0, \infty)$, and

$$
\sup _{x \in(0, \infty)} \varphi(x)\left(\int_{0}^{\infty} \frac{|f(y)|}{u(x)+u(y)} d y\right),
$$


when $q>0$; here $u$ and $\varphi$ are weights satisfying certain conditions (see Sections 2 and 3 below). Let us recall that, in a very general form, the Hilbert norm of a function $f$ is the quantity

$$
\left(\int_{0}^{\infty} \varphi(x)\left(\int_{0}^{\infty} \frac{|f(y)|}{u(x)+u(y)} d y\right)^{q} \varphi(x) d x\right)^{\frac{1}{q}}
$$

when $q \in(0, \infty)$, and

$$
\sup _{x \in(0, \infty)} \varphi(x)\left(\int_{0}^{\infty} \frac{|f(y)|}{u(x)+u(y)} d y\right),
$$

when $q>0$; here $u$ and $\varphi$ are weights satisfying certain conditions (see Sections 2 and 3 below).

We point out three of the major applications of our results. In Sections 4 and 5 we establish integral necessary and sufficient conditions for the embeddings $\Gamma^{p}(v) \hookrightarrow \Lambda^{q}(w)$ and $\Gamma^{p}(v) \hookrightarrow \Gamma^{q}(w)$, respectively. Finally, in Section 6, we give a precise integral characterization of the associate space of $\Gamma^{p}(v)$. The results on spaces $\Gamma^{p}(v)$ can be extended to the more general context of $K$-spaces (defined through the Peetre $K$-functional).

Everywhere below, $u, v$ and $w$ are weights. We shall throughout denote $U(t)=\int_{0}^{t} u(s) d s, V(t)=\int_{0}^{t} v(s) d s$ and $W(t)=\int_{0}^{t} w(s) d s$ for $t \in(0, \infty)$. By $A \lesssim B$ we mean that $A \leq C B$ with some positive $C$ independent of appropriate quantities. If $A \lesssim B$ and $B \lesssim A$, we write $A \approx B$. We say that two functions $f, g$ are equivalent on $(0, \infty)$ if there exists a constant $C>0$ such that

$$
C^{-1} f(t) \leq g(t) \leq C f(t) \text { for all } t \in(0, \infty) .
$$

\section{General anti-discretization theorems}

We start with some basic definitions. We follow [15], [25] and [16].

Definition 2.1. Let $\left\{a_{k}\right\}$ be a sequence of positive real numbers. We say that $\left\{a_{k}\right\}$ is strongly increasing or strongly decreasing and write $\left\{a_{k}\right\} \uparrow$ or $\left\{a_{k}\right\} \downarrow$ when

$$
\inf _{k \in \mathbb{Z}} \frac{a_{k+1}}{a_{k}}>1 \text { or } \sup _{k \in \mathbb{Z}} \frac{a_{k+1}}{a_{k}}<1,
$$

respectively.

Definition 2.2. Let $\varphi$ be a continuous strictly increasing function on $[0, \infty)$ such that $\varphi(0)=0$ and $\lim _{t \rightarrow \infty} \varphi(t)=\infty$. Then we say that $\varphi$ is admissible. 
Let $\varphi$ be an admissible function. We say that a function $h$ is $\varphi$-quasiconcave if $h$ is equivalent to a non-decreasing function on $[0, \infty)$ and $\frac{h}{\varphi}$ is equivalent to a non-increasing function on $(0, \infty)$. We say that a $\varphi$-quasiconcave function $h$ is non-degenerate if

$$
\lim _{t \rightarrow 0+} h(t)=\lim _{t \rightarrow \infty} \frac{1}{h(t)}=\lim _{t \rightarrow \infty} \frac{h(t)}{\varphi(t)}=\lim _{t \rightarrow 0+} \frac{\varphi(t)}{h(t)}=0 .
$$

The family of non-degenerate $\varphi$-quasiconcave functions will be denoted by $\Omega_{\varphi}$.

We say that $h$ is quasiconcave when $h \in \Omega_{\varphi}$ with $\varphi(t)=t$.

Remarks 2.3. (i) It will be useful to note that

$$
h \in \Omega_{\varphi} \Longleftrightarrow \frac{\varphi}{h} \in \Omega_{\varphi}
$$

(ii) Some authors add the restriction $h(t)=0$ if and only if $t=0$ to the definition of a quasiconcave function. However, the only difference is that our definition recognizes the zero function as quasiconcave.

(iii) Note that any non-degenerate $\varphi$-quasiconcave function is necessarily continuous on $[0, \infty)$.

Definition 2.4. Assume that $\varphi$ is admissible and $h \in \Omega_{\varphi}$. We say that $\left\{\mu_{k}\right\}_{k \in \mathbb{Z}}$ is a discretizing sequence for $h$ with respect to $\varphi$ if

(i) $\mu_{0}=1$ and $\varphi\left(\mu_{k}\right) \uparrow$;

(ii) $h\left(\mu_{k}\right) \uparrow \uparrow$ and $\frac{h\left(\mu_{k}\right)}{\varphi\left(\mu_{k}\right)} \downarrow$;

(iii) there is a decomposition $\mathbb{Z}=\mathbb{Z}_{1} \cup \mathbb{Z}_{2}$ such that $\mathbb{Z}_{1} \cap \mathbb{Z}_{2}=\emptyset$ and, for every $t \in\left[\mu_{k}, \mu_{k+1}\right]$,

$$
h\left(\mu_{k}\right) \approx h(t) \quad \text { if } \quad k \in \mathbb{Z}_{1},
$$

$$
\frac{h\left(\mu_{k}\right)}{\varphi\left(\mu_{k}\right)} \approx \frac{h(t)}{\varphi(t)} \quad \text { if } \quad k \in \mathbb{Z}_{2} .
$$

Remark 2.5. In [16], a special case $\varphi(t)=t$ is treated.

Examples 2.6. (i) Let $\varphi(t)=t^{\alpha}, \alpha>0$. Then we recover the situation which has been in one way or another treated by several authors (cf. e.g. [5], [22] or $[\mathbf{4}]$ ).

(ii) When $u$ is a positive function on $[0, \infty)$ such that $\int_{0}^{\infty} u(s) d s=$ $\infty$ and $f$ is a locally integrable function, then $K_{u}(f, t) \in \Omega_{U}$, where $K_{u}(f, t)=\int_{0}^{t} f^{*}(s) u(s) d s$ and $U(t)=\int_{0}^{t} u(s) d s$. The operator $K_{u}$ is a particular case of the well-known Peetre $K$-functional. 
(iii) Recently, a considerable attention is being paid to the Hardy operators involving suprema such as $R_{u}$, defined at a locally integrable function $f$ by

$$
\left(R_{u} f\right)(t)=\sup _{s \in[t, \infty)} u(s) \int_{0}^{s} f^{*}(y) d y, \quad t \in(0, \infty),
$$

where $u$ is a given weight on $(0, \infty)$ (see for example $[\mathbf{1 1}],[\mathbf{1 3}]$ or $[\mathbf{1 9}]$ ). If moreover $u$ is such that the function $\varphi$, defined by

$$
\varphi(t)=\left(\sup _{s \in[t, \infty)} u(s)\right)^{-1}, \quad t \in(0, \infty),
$$

is admissible, then, for any $f, \varphi R_{u} f$ is $\varphi$-quasiconcave. Indeed, this follows from the readily verified relation

$$
\varphi(t)\left(R_{u} f\right)(t) \approx \sup _{s \in(0, \infty)} \frac{\varphi(t)}{\varphi(s)+\varphi(t)} \int_{0}^{s} f^{*}(y) d y, \quad t \in(0, \infty) .
$$

(iv) Let $0<p_{0}<p_{1}<\infty, 0<q_{0} \leq q_{1}<\infty$ and let

$$
m=\frac{\frac{1}{q_{0}}-\frac{1}{q_{1}}}{\frac{1}{p_{0}}-\frac{1}{p_{1}}}
$$

Then the Calderón operator (cf. [3, Chapter 3, Definition 5.1])

$$
\left(S f^{*}\right)(t)=t^{-\frac{1}{q_{0}}} \int_{0}^{t^{m}} s^{\frac{1}{p_{0}}-1} f^{*}(s) d s+t^{-\frac{1}{q_{1}}} \int_{t^{m}}^{\infty} s^{\frac{1}{p_{1}}-1} f^{*}(s) d s
$$

satisfies, for any fixed (appropriate) $f,\left(S f^{*}\right)(t) t^{\frac{1}{q_{0}}} \in \Omega_{\varphi}$, where $\varphi(t)=$ $t^{\frac{1}{q_{0}}-\frac{1}{q_{1}}}$.

Lemma 2.7. Let $\varphi$ be an admissible function on $[0, \infty)$, let $h \in \Omega_{\varphi}$ and let $a>1$. We define the sequence $\left\{\mu_{k}\right\}$ by $\mu_{0}=1$ and

$$
\mu_{k+1}=\inf \left\{t ; \min \left\{\frac{h(t)}{h\left(\mu_{k}\right)}, \frac{h\left(\mu_{k}\right) \varphi(t)}{\varphi\left(\mu_{k}\right) h(t)}\right\}=a\right\}, \text { when } k \geq 0 ;
$$

(2.6) $\mu_{k-1}=\inf \left\{t ; \min \left\{\frac{h\left(\mu_{k}\right)}{h(t)}, \frac{h(t) \varphi\left(\mu_{k}\right)}{\varphi(t) h\left(\mu_{k}\right)}\right\}=a\right\}$, when $k \leq 0$.

Then $\left\{\mu_{k}\right\}$ is a discretizing sequence for $h$ with respect to $\varphi$.

Proof: We have to show that the properties (i), (ii), (iii) from Definition 2.4 are satisfied. Set

$$
\mathbb{Z}_{1}=\left\{k \in \mathbb{Z} ; a h\left(\mu_{k}\right)=h\left(\mu_{k+1}\right)\right\}, \quad \mathbb{Z}_{2}=\mathbb{Z} \backslash \mathbb{Z}_{1} .
$$


Then

$$
\frac{h\left(\mu_{k}\right)}{\varphi\left(\mu_{k}\right)}=a \frac{h\left(\mu_{k+1}\right)}{\varphi\left(\mu_{k+1}\right)} \quad \text { for } k \in \mathbb{Z}_{2} .
$$

Since $h \in \Omega_{\varphi}$, it follows from (2.5) and (2.6) that $\mu_{k+1}>\mu_{k}$ for every $k \in \mathbb{Z}$. Hence, using also (2.5) and (2.6), we get for every $k \in \mathbb{Z}$,

$$
\frac{h\left(\mu_{k}\right)}{\varphi\left(\mu_{k}\right)} \geq a \frac{h\left(\mu_{k+1}\right)}{\varphi\left(\mu_{k+1}\right)} \geq a \frac{h\left(\mu_{k}\right)}{\varphi\left(\mu_{k+1}\right)}
$$

and therefore, for $k \in \mathbb{Z}$,

$$
\frac{\varphi\left(\mu_{k+1}\right)}{\varphi\left(\mu_{k}\right)} \geq a>1
$$

This shows that $\varphi\left(\mu_{k}\right) \uparrow$. Next, by (2.5) and (2.6), we have, for $k \in \mathbb{Z}$,

$$
\frac{h\left(\mu_{k+1}\right)}{h\left(\mu_{k}\right)} \geq a>1, \quad \text { and } \quad \frac{h\left(\mu_{k+1}\right)}{\varphi\left(\mu_{k+1}\right)} \frac{\varphi\left(\mu_{k}\right)}{h\left(\mu_{k}\right)} \leq \frac{1}{a}<1,
$$

so $h\left(\mu_{k}\right) \uparrow$ and $\frac{h\left(\mu_{k}\right)}{\varphi\left(\mu_{k}\right)} \downarrow$.

Finally, $h \in \Omega_{\varphi}$, whence, by (2.7), for $t \in\left[\mu_{k}, \mu_{k+1}\right]$,

$$
\begin{cases}h(t) \leq h\left(\mu_{k+1}\right)=a h\left(\mu_{k}\right) \leq a h(t) & \text { when } k \in \mathbb{Z}_{1} ; \\ \frac{h(t)}{\varphi(t)} \leq \frac{h\left(\mu_{k}\right)}{\varphi\left(\mu_{k}\right)}=a \frac{h\left(\mu_{k+1}\right)}{\varphi\left(\mu_{k+1}\right)} \leq a \frac{h(t)}{\varphi(t)} & \text { when } k \in \mathbb{Z}_{2},\end{cases}
$$

showing (iii).

Lemma 2.8. Let $\varphi$ be an admissible function. Then the following statements are equivalent:

(i) $h \in \Omega_{\varphi}$;

(ii) there exists a non-increasing function $\psi$ such that

$$
h(t) \approx \int_{0}^{t} \psi(s) d \varphi(s), \quad t \in(0, \infty)
$$

(with the Lebesgue-Stieltjes integral on the right);

(iii) there is a non-negative Borel measure $\eta$ on $[0, \infty)$ such that

$$
h(t) \approx \int_{[0, t]} \varphi(s) d \eta(s)+\varphi(t) \int_{[t, \infty)} d \eta(s), \quad t \in(0, \infty) .
$$

Proof: (i) $\Rightarrow$ (ii) Let $h \in \Omega_{\varphi}$. Then $h$ is a non-degenerate $\varphi$-quasiconcave function, and so $h\left(\varphi^{-1}\right)$ is quasi-concave with

$$
\lim _{t \rightarrow 0+} h\left(\varphi^{-1}\right)(t)=\lim _{t \rightarrow \infty} \frac{h\left(\varphi^{-1}\right)(t)}{t}=0 .
$$


Thus, there exists a non-increasing function $\theta$ on $(0, \infty)$ such that

$$
h\left(\varphi^{-1}\right)(t) \approx \int_{0}^{t} \theta(s) d s, \quad t \in(0, \infty) .
$$

Hence,

$$
h(t) \approx \int_{0}^{\varphi(t)} \theta(s) d s=\int_{0}^{t} \theta(\varphi(s)) d \varphi(s), \quad t \in(0, \infty) .
$$

Denoting $\psi=\theta \circ \varphi$, we get (ii).

(ii) $\Rightarrow$ (iii) Since $\theta$ is non-increasing, it follows that $\psi$ is non-increasing, too. Integrating by parts, we get

$$
h(t) \approx \psi(t) \varphi(t)-\int_{0}^{t} \varphi(s) d \psi(s), \quad t \in(0, \infty),
$$

and, to get (iii), it suffices to denote $d \eta=d(-\psi)$.

(iii) $\Rightarrow$ (i) Assume that $h$ can be represented as in (2.9). Then, denoting $d \nu(s)=\varphi(s) d \eta(s)$, we have

$$
h(t) \approx \varphi(t) \int_{[0, \infty)} \frac{d \nu(s)}{\varphi(s)+\varphi(t)}, \quad t \in(0, \infty) .
$$

Now, the monotonicity properties required in order to verify (i) are obvious.

Definition 2.9. Let $\varphi$ be an admissible function and let $\nu$ be a nonnegative Borel measure on $[0, \infty)$. We say that the function $h$ defined as

$$
h(t)=\varphi(t) \int_{[0, \infty)} \frac{d \nu(s)}{\varphi(s)+\varphi(t)}, \quad t \in(0, \infty),
$$

is the fundamental function of the measure $\nu$ with respect to $\varphi$. We will also say that $\nu$ is a representation measure of $h$ with respect to $\varphi$.

We say that $\nu$ is non-degenerate if the following conditions are satisfied for every $t \in(0, \infty)$ :

$\int_{[0, \infty)} \frac{d \nu(s)}{\varphi(s)+\varphi(t)}<\infty, \quad t \in(0, \infty), \quad \int_{[0,1]} \frac{d \nu(s)}{\varphi(s)}=\int_{[1, \infty)} d \nu(s)=\infty$.

Remark 2.10. (i) Let $\varphi$ be an admissible function and let $\nu$ be a nonnegative non-degenerate Borel measure on $[0, \infty)$. Let $h$ be the fundamental function of $\nu$ with respect to $\varphi$. Then

$$
h(t) \approx \int_{0}^{t} \int_{[s, \infty)} \frac{d \nu(y)}{\varphi(y)} d \varphi(s), \quad t \in(0, \infty),
$$


and also

$$
h(t) \approx \int_{[0, t]} d \nu(s)+\varphi(t) \int_{[t, \infty)} \frac{d \nu(s)}{\varphi(s)}, \quad t \in(0, \infty) .
$$

Moreover, $h \in \Omega_{\varphi}$. In particular, by Lemma 2.7, there always exists a discretizing sequence for $h$ with respect to $\varphi$.

Indeed, (2.12) follows immediately from (2.11) and the Fubini theorem, while (2.13) is an immediate consequence of (2.11) and the monotonicity of $\varphi$. From (2.12) it is clear that $h$ is non-decreasing and also that $\frac{h}{\varphi}$ is non-increasing because it is an integral mean of a nonincreasing function. Finally, the non-degeneracy requirements in (2.1) follow from (2.12) and (2.13) combined with the Monotone Convergence Theorem.

(ii) Conversely, if $\varphi$ is an admissible function and $h \in \Omega_{\varphi}$, then there exists a representation measure $\nu$. This follows from Remark 2.10 (i) and Lemma 2.8; indeed, we can put $d \nu=\varphi d \eta$.

Theorem 2.11. Let $p, q, r \in(0, \infty)$. Let $u$ be an admissible function. Let $\nu$ be a non-negative non-degenerate Borel measure on $[0, \infty)$, and let $h$ be the fundamental function of $\nu$ with respect to $u^{q}$. Let $\sigma \in \Omega_{u^{p}}$. Let $\left\{\mu_{k}\right\}$ be a discretizing sequence for $h$ with respect to $u^{q}$. Then

$$
\int_{[0, \infty)} \frac{h(t)^{\frac{r}{q}-1}}{\sigma(t)^{\frac{r}{p}}} d \nu(t) \approx \sum_{k \in \mathbb{Z}} \frac{h\left(\mu_{k}\right)^{\frac{r}{q}}}{\sigma\left(\mu_{k}\right)^{\frac{r}{p}}} .
$$

Proof: Let $a>2$, and let $\mathbb{Z}_{1}, \mathbb{Z}_{2}$ be defined by (2.7) (with $\varphi=u^{q}$ ). Then

$$
\int_{[0, \infty)} \frac{h(t)^{\frac{r}{q}-1}}{\sigma(t)^{\frac{r}{p}}} d \nu(t)=\sum_{k \in \mathbb{Z}} \int_{\left[\mu_{k}, \mu_{k+1}\right)} \frac{h(t)^{\frac{r}{q}-1}}{\sigma(t)^{\frac{r}{p}}} d \nu(t)=\sum_{k \in \mathbb{Z}_{1}}+\sum_{k \in \mathbb{Z}_{2}}=\mathrm{I}+\mathrm{II},
$$

say. For $k \in \mathbb{Z}_{1}, h\left(\mu_{k+1}\right)=a h\left(\mu_{k}\right)$, and

$$
\int_{\left[\mu_{k}, \mu_{k+1}\right)} d \nu(t) \lesssim h\left(\mu_{k+1}\right) \lesssim h\left(\mu_{k}\right) .
$$

Thus, since $\sigma$ is equivalent to a non-decreasing function, we get from (2.3)

$$
\mathrm{I} \lesssim \sum_{k \in \mathbb{Z}_{1}} \frac{h\left(\mu_{k}\right)^{\frac{r}{q}-1}}{\sigma\left(\mu_{k}\right)^{\frac{r}{p}}} \int_{\left[\mu_{k}, \mu_{k+1}\right)} d \nu(t) \lesssim \sum_{k \in \mathbb{Z}_{1}} \frac{h\left(\mu_{k}\right)^{\frac{r}{q}-1}}{\sigma\left(\mu_{k}\right)^{\frac{r}{p}}} h\left(\mu_{k}\right) \lesssim \sum_{k \in \mathbb{Z}_{1}} \frac{h\left(\mu_{k}\right)^{\frac{r}{q}}}{\sigma\left(\mu_{k}\right)^{\frac{r}{p}}} .
$$

For $k \in \mathbb{Z}_{2}$, by (2.8) we have

$$
a \frac{h\left(\mu_{k+1}\right)}{u\left(\mu_{k+1}\right)^{q}}=\frac{h\left(\mu_{k}\right)}{u\left(\mu_{k}\right)^{q}},
$$


and therefore

$$
\int_{\left[\mu_{k}, \mu_{k+1}\right)} u^{-q}(t) d \nu(t) \lesssim \frac{h\left(\mu_{k}\right)}{u^{q}\left(\mu_{k}\right)} \lesssim \frac{h\left(\mu_{k+1}\right)}{u^{q}\left(\mu_{k+1}\right)} .
$$

Since $\sigma u^{-p}$ is decreasing, using (2.4) and (2.8) we obtain

$$
\begin{aligned}
\mathrm{II} & =\sum_{k \in \mathbb{Z}_{2}} \int_{\left[\mu_{k}, \mu_{k+1}\right)} \frac{h(t)^{\frac{r}{q}-1}}{\sigma(t)^{\frac{r}{p}}} d \nu(t) \\
& =\sum_{k \in \mathbb{Z}_{2}} \int_{\left[\mu_{k}, \mu_{k+1}\right)} \frac{h(t)^{\frac{r}{q}-1}}{u(t)^{r}} \frac{u(t)^{r}}{\sigma(t)^{\frac{r}{p}}} d \nu(t) \\
& \leq \sum_{k \in \mathbb{Z}_{2}} \frac{u\left(\mu_{k+1}\right)^{r}}{\sigma\left(\mu_{k+1}\right)^{\frac{r}{p}}} \int_{\left[\mu_{k}, \mu_{k+1}\right)} \frac{h(t)^{\frac{r}{q}-1}}{u(t)^{r}} d \nu(t) \\
& \approx \sum_{k \in \mathbb{Z}_{2}} \frac{h\left(\mu_{k+1}\right)^{\frac{r}{q}-1}}{\sigma\left(\mu_{k+1}\right)^{\frac{r}{p}}} u\left(\mu_{k+1}\right)^{q} \int_{\left[\mu_{k}, \mu_{k+1}\right)} u(t)^{-q} d \nu(t) \\
& \lesssim \sum_{k \in \mathbb{Z}} \frac{h\left(\mu_{k+1}\right)^{\frac{r}{q}}}{\sigma\left(\mu_{k+1}\right)^{\frac{r}{p}}} .
\end{aligned}
$$

This shows the upper bound.

To prove the converse, let $\lambda_{k} \in\left[\mu_{k-1}, \mu_{k}\right)$ be such that $h\left(\mu_{k}\right)=a h\left(\lambda_{k}\right)$ and $\gamma_{k} \in\left(\mu_{k}, \mu_{k+1}\right]$ be such that

$$
a \frac{h\left(\gamma_{k}\right)}{u\left(\gamma_{k}\right)^{q}}=\frac{h\left(\mu_{k}\right)}{u\left(\mu_{k}\right)^{q}} .
$$

Therefore, we have (using that $\sigma u^{-p}$ is equivalent to a non-increasing function)

$$
\int_{\left[\mu_{k}, \mu_{k+1}\right)} \frac{h(t)^{\frac{r}{q}-1}}{\sigma(t)^{\frac{r}{p}}} d \nu(t) \gtrsim \frac{h\left(\mu_{k}\right)^{\frac{r}{q}-1}}{\sigma\left(\mu_{k}\right)^{\frac{r}{p}}} u\left(\mu_{k}\right)^{q} \int_{\left[\mu_{k}, \gamma_{k}\right)} u(t)^{-q} d \nu(t)
$$

and, using that $\sigma$ is equivalent to a non-decreasing function and (2.3),

$$
\int_{\left[\mu_{k-1}, \mu_{k}\right)} \frac{h(t)^{\frac{r}{q}-1}}{\sigma(t)^{\frac{r}{p}}} d \nu(t) \gtrsim \frac{h\left(\mu_{k}\right)^{\frac{r}{q}-1}}{\sigma\left(\mu_{k}\right)^{\frac{r}{p}}} \int_{\left[\lambda_{k}, \mu_{k}\right)} d \nu(t) .
$$


Combining the last two estimates we get

$$
\begin{aligned}
& \int_{\left[\mu_{k-1}, \mu_{k+1}\right)} \frac{h(t)^{\frac{r}{q}-1}}{\sigma(t)^{\frac{r}{p}}} d \nu(t) \\
& \gtrsim \frac{h\left(\mu_{k}\right)^{\frac{r}{q}-1}}{\sigma\left(\mu_{k}\right)^{\frac{r}{p}}}\left[\int_{\left[0, \mu_{k}\right]} d \nu(t)+u\left(\mu_{k}\right)^{q} \int_{\left[\mu_{k}, \infty\right)} u(t)^{-q} d \nu(t)\right. \\
&\left.\quad-\int_{\left[0, \lambda_{k}\right]} d \nu(t)-u\left(\mu_{k}\right)^{q} \int_{\left[\gamma_{k}, \infty\right)} u(t)^{-q} d \nu(t)\right] \\
& \gtrsim \frac{h\left(\mu_{k}\right)^{\frac{r}{q}-1}}{\sigma\left(\mu_{k}\right)^{\frac{r}{p}}}\left[h\left(\mu_{k}\right)-h\left(\lambda_{k}\right)-\frac{h\left(\gamma_{k}\right) u\left(\mu_{k}\right)^{q}}{u\left(\gamma_{k}\right)^{q}}\right] \\
&=\frac{h\left(\mu_{k}\right)^{\frac{r}{q}-1}}{\sigma\left(\mu_{k}\right)^{\frac{r}{p}}}\left[h\left(\mu_{k}\right)-\frac{2}{a} h\left(\mu_{k}\right)\right] \\
&=\left(1-\frac{2}{a}\right) \frac{h\left(\mu_{k}\right)^{\frac{r}{q}}}{\sigma\left(\mu_{k}\right)^{\frac{r}{p}}},
\end{aligned}
$$

from which we obtain

$$
\int_{[0, \infty)} \frac{h(t)^{\frac{r}{q}-1}}{\sigma(t)^{\frac{r}{p}}} d \nu(t) \approx \sum_{k \in \mathbb{Z}} \int_{\left[\mu_{k-1}, \mu_{k+1}\right)} \frac{h(t)^{\frac{r}{q}-1}}{\sigma(t)^{\frac{r}{p}}} d \nu(t) \gtrsim \sum_{k \in \mathbb{Z}} \frac{h\left(\mu_{k}\right)^{\frac{r}{q}}}{\sigma\left(\mu_{k}\right)^{\frac{r}{p}}},
$$

proving the lower bound.

The key problem for the application of the preceding theorem is, given $h$, to find a non-negative representation measure $\nu$. A simple sufficient condition for this to happen is formulated in the next example.

Example 2.12. Let $p, q, r, u$ and $\sigma$ be as in Theorem 2.11 and assume further that $h \geq 0$ is a non-decreasing function on $[0, \infty)$ such that $h^{\prime}$ and $\left(u^{q}\right)^{\prime}$ exist and

$$
\frac{h^{\prime}}{\left(u^{q}\right)^{\prime}} \quad \text { is nonincreasing and continuous. }
$$

Set

$$
\Phi(s)=-\frac{h^{\prime}(s)}{\left(u^{q}\right)^{\prime}(s)}, \quad s \in(0, \infty) .
$$


By differentiation,

$$
h(t)=\int_{0}^{t} u(s)^{q} d \Phi(s)+u(t)^{q} \int_{t}^{\infty} d \Phi(s), \quad t \in(0, \infty) .
$$

Then, $u^{q} d \Phi$ is a positive measure, and $h \in \Omega_{u^{q}}$ by Remark 2.10 (i). Furthermore, let $\left\{\mu_{k}\right\}$ be a discretizing sequence for $h$ with respect to $u^{q}$. Then, by Theorem 2.11,

$$
\int_{0}^{\infty} \frac{h(t)^{\frac{r}{q}-1}}{\sigma(t)^{\frac{r}{p}}} u(t)^{q} d \Phi(t) \approx \sum_{k \in \mathbb{Z}} \frac{h\left(\mu_{k}\right)^{\frac{r}{q}}}{\sigma\left(\mu_{k}\right)^{\frac{r}{p}}} .
$$

In the special case we get the following discretization formula for the Lebesgue norm.

Corollary 2.13. Let $q \in(0, \infty)$, let $u$ be an admissible function, let $f \in \Omega_{u}$, let $\nu$ be a non-negative non-degenerate Borel measure on $[0, \infty)$ and let $h$ be the fundamental function of $\nu$ with respect to $u^{q}$. Let $\left\{\mu_{k}\right\}$ be a discretizing sequence for $h$ with respect to $u^{q}$. Then

$$
\left(\int_{[0, \infty)}\left(\frac{f(t)}{u(t)}\right)^{q} d \nu(t)\right)^{\frac{1}{q}} \approx\left(\sum_{k \in \mathbb{Z}}\left(\frac{f\left(\mu_{k}\right)}{u\left(\mu_{k}\right)}\right)^{q} h\left(\mu_{k}\right)\right)^{\frac{1}{q}} .
$$

Proof: We apply Theorem 2.11 with the following parameters: $r=q$, $p=1$, and $\sigma=\frac{u}{f}$. It remains to observe that, by (2.2), $f \in \Omega_{u}$ is equivalent to $\sigma \in \Omega_{u}$.

Theorem 2.14. Let $\varphi$ be an admissible function, let $\nu$ be a non-negative non-degenerate Borel measure on $[0, \infty)$, and let $h$ be the fundamental function of $\nu$ with respect to $\varphi$. Set

$$
d \theta(t)=\frac{\varphi(t) \int_{[0, t]} d \nu(y) \int_{[t, \infty)} \frac{d \nu(s)}{\varphi(s)}}{h^{3}(t)} d \varphi(t), \quad t \in(0, \infty) .
$$

Then $\frac{\varphi}{h}$ is equivalent to the fundamental function of $\theta$ with respect to $\varphi$.

Proof: We denote

$$
\mathrm{I}=\int_{0}^{t} \frac{\varphi(s) \int_{[0, s]} d \nu(y) \int_{[s, \infty)} \frac{d \nu(\tau)}{\varphi(\tau)}}{h^{3}(s)} d \varphi(s), \quad t \in(0, \infty),
$$

and

$$
\mathrm{II}=\varphi(t) \int_{t}^{\infty} \frac{\int_{[0, s]} d \nu(y) \int_{[s, \infty)} \frac{d \nu(\tau)}{\varphi(\tau)}}{h^{3}(s)} d \varphi(s), \quad t \in(0, \infty),
$$


and claim that

$$
\frac{\varphi(t)}{h(t)} \approx \mathrm{I}+\mathrm{II}, \quad t \in(0, \infty)
$$

We start with proving the lower bound. First note that, by (2.13),

$$
\varphi(s) \int_{[s, \infty)} \frac{d \nu(y)}{\varphi(y)} \lesssim h(s), \quad s \in(0, \infty)
$$

Therefore, for $t \in(0, \infty)$,

$$
\begin{aligned}
\mathrm{I} & \lesssim \int_{0}^{t} \frac{\int_{[0, s]} d \nu(y)}{\left[\int_{[0, s]} d \nu(y)+\varphi(s) \int_{[s, \infty)} \frac{d \nu(\tau)}{\varphi(\tau)}\right]^{2}} d \varphi(s) \\
& =\int_{0}^{t} \frac{\varphi(s)^{-2} \int_{[0, s]} d \nu(y)}{\left[\varphi(s)^{-1} \int_{[0, s]} d \nu(y)+\int_{[s, \infty)} \frac{d \nu(\tau)}{\varphi(\tau)}\right]^{2}} d \varphi(s) \\
& =\frac{\varphi(t)}{h(t)}
\end{aligned}
$$

Similarly, using the estimate

$$
\int_{[0, s]} d \nu(y) \lesssim h(s), \quad s \in(0, \infty)
$$

which, once again, follows from (2.13), we have

$$
\mathrm{II} \lesssim \varphi(t) \int_{t}^{\infty} \frac{\int_{[s, \infty)} \frac{d \nu(\tau)}{\varphi(\tau)}}{\left[\int_{[0, s]} d \nu(y)+\varphi(s) \int_{[s, \infty)} \frac{d \nu(\tau)}{\varphi(\tau)}\right]^{2}} d \varphi(s)=\frac{\varphi(t)}{h(t)}, \quad t \in(0, \infty)
$$

Thus, finally,

$$
\mathrm{I}+\mathrm{II} \lesssim \frac{\varphi(t)}{h(t)}, \quad t \in(0, \infty)
$$


Conversely, observe that, for $t \in(0, \infty)$,

$$
\begin{aligned}
\mathrm{I} & \gtrsim \int_{[t, \infty)} \frac{d \nu(\tau)}{\varphi(\tau)} \int_{0}^{t} \frac{\int_{[0, s]} d \nu(y) \varphi(s)^{-2}}{\left[\varphi(s)^{-1} \int_{[0, s]} d \nu(y)+\int_{[s, \infty)} \frac{d \nu(\tau)}{\varphi(\tau)}\right]^{3}} d \varphi(s) \\
& \gtrsim \frac{\int_{[t, \infty)} \frac{d \nu(\tau)}{\varphi(\tau)}}{\left[\varphi(t)^{-1} \int_{[0, t]} d \nu(y)+\int_{[t, \infty)} \frac{d \nu(\tau)}{\varphi(\tau)}\right]^{2}} \\
& \approx \frac{\varphi(t)^{2} \int_{[t, \infty)} \frac{d \nu(\tau)}{\varphi(\tau)}}{h(t)^{2}},
\end{aligned}
$$

and, similarly,

$$
\begin{aligned}
\mathrm{II} & \gtrsim \varphi(t) \int_{[0, t]} d \nu(y) \int_{t}^{\infty} \frac{\int_{[s, \infty)} \frac{d \nu(\tau)}{\varphi(\tau)}}{\left[\int_{[0, s]} d \nu(y)+\varphi(s) \int_{[s, \infty)} \frac{d \nu(\tau)}{\varphi(\tau)}\right]^{3}} d \varphi(s) \\
& \gtrsim \frac{\varphi(t) \int_{[0, t]} d \nu(y)}{h(t)^{2}} .
\end{aligned}
$$

Now, obviously,

$$
\mathrm{I}+\mathrm{II} \gtrsim \frac{\varphi(t) \int_{[0, t]} d \nu(y)+\varphi(t)^{2} \int_{[t, \infty)} \frac{d \nu(\tau)}{\varphi(\tau)}}{h(t)^{2}} \gtrsim \frac{\varphi(t)}{h(t)}, \quad t \in(0, \infty) .
$$

Theorem 2.15. Let $p, q, r \in(0, \infty)$. Let $u$ be an admissible function. Let $f \in \Omega_{u^{p}}$. Let $\nu$ be a non-negative non-degenerate Borel measure on $[0, \infty)$ and let $g$ be the fundamental function of $\nu$ with respect to $u^{q}$. Let $\left\{\mu_{k}\right\}$ be a discretizing sequence of $g$ with respect to $u^{q}$. Then

$$
\int_{0}^{\infty} \frac{f(t)^{\frac{r}{p}} \int_{[0, t]} d \nu(s) \int_{[t, \infty)} u(y)^{-q} d \nu(y)}{g(t)^{\frac{r}{q}+2}} d\left(u^{q}\right)(t) \approx \sum_{k \in \mathbb{Z}} \frac{f\left(\mu_{k}\right)^{\frac{r}{p}}}{g\left(\mu_{k}\right)^{\frac{r}{q}}}
$$

Proof: Set

$$
d \theta(t)=\frac{u(t)^{q} \int_{[0, t)} d \nu(s) \int_{[t, \infty)} \frac{d \nu(y)}{u(y)^{q}}}{g^{3}(t)} d\left(u^{q}\right)(t), \quad t \in(0, \infty) .
$$

Then, by Theorem 2.14, $\frac{u^{q}}{g}$ is equivalent to the fundamental function of $\theta$ with respect to $u^{q}$. Note that, by (2.2), $f \in \Omega_{u^{p}}$ is equivalent to 
$\frac{u^{p}}{f} \in \Omega_{u^{p}}$. Therefore, by Theorem 2.11, applied to $h=\frac{u^{q}}{g}, \nu=\theta$ and $\sigma=\frac{u^{p}}{f}$, we obtain

$$
\int_{0}^{\infty} \frac{\left[\frac{u(t)^{q}}{g(t)}\right]^{\frac{r}{q}-1}}{\left[\frac{u(t)^{p}}{f(t)}\right]^{\frac{r}{p}}} d \theta(t) \approx \sum_{k \in \mathbb{Z}} \frac{\left[\frac{u\left(\mu_{k}\right)^{q}}{g\left(\mu_{k}\right)}\right]^{\frac{r}{q}}}{\left[\frac{u\left(\mu_{k}\right)^{p}}{f\left(\mu_{k}\right)}\right]^{\frac{r}{p}}}=\sum_{k \in \mathbb{Z}} \frac{f\left(\mu_{k}\right)^{\frac{r}{p}}}{g\left(\mu_{k}\right)^{\frac{r}{q}}} .
$$

Since the integrals on the left-hand sides of (2.14) and (2.15) coincide, the assertion follows.

\section{Discretization of the Hilbert norm}

We begin by quoting some known results. For our first lemma, see for example [16].

Lemma 3.1. Let $\left\{a_{k}\right\}_{k \in \mathbb{Z}},\left\{\sigma_{k}\right\}_{k \in \mathbb{Z}}$, and $\left\{\tau_{k}\right\}_{k \in \mathbb{Z}}$ be sequences of nonnegative numbers. Let $p \in(0, \infty)$.

(i) If $\sigma_{k} \uparrow$, then

$$
\left\{\sum_{k \in \mathbb{Z}}\left[\sum_{m=k}^{\infty} a_{m}\right]^{p} \sigma_{k}^{p}\right\}^{\frac{1}{p}} \approx\left\{\sum_{m \in \mathbb{Z}}\left(a_{m} \sigma_{m}\right)^{p}\right\}^{\frac{1}{p}} .
$$

(ii) If $\tau_{k} \downarrow$, then

$$
\left\{\sum_{k \in \mathbb{Z}}\left[\sum_{m=-\infty}^{k} a_{m}\right]^{p} \tau_{k}^{p}\right\}^{\frac{1}{p}} \approx\left\{\sum_{m \in \mathbb{Z}}\left(a_{m} \tau_{m}\right)^{p}\right\}^{\frac{1}{p}} .
$$

The following three lemmas are elementary and most likely well known. For example, they can be proved by an argument similar to that used in the proof of [16, Proposition 2.1].

Lemma 3.2. Let $\left\{a_{k}\right\}_{k \in \mathbb{Z}},\left\{\sigma_{k}\right\}_{k \in \mathbb{Z}}$, and $\left\{\tau_{k}\right\}_{k \in \mathbb{Z}}$ be sequences of nonnegative numbers.

(i) If $\sigma_{k} \uparrow$, then

$$
\sum_{k \in \mathbb{Z}}\left(\sup _{m \geq k} a_{m}\right) \sigma_{k} \approx \sum_{m \in \mathbb{Z}} a_{m} \sigma_{m} .
$$

(ii) If $\tau_{k} \downarrow$, then

$$
\sum_{k \in \mathbb{Z}}\left(\sup _{m \leq k} a_{m}\right) \tau_{k} \approx \sum_{m \in \mathbb{Z}} a_{m} \tau_{m} .
$$


Lemma 3.3. Let $\left\{a_{k}\right\}_{k \in \mathbb{Z}},\left\{\sigma_{k}\right\}_{k \in \mathbb{Z}}$, and $\left\{\tau_{k}\right\}_{k \in \mathbb{Z}}$ be sequences of nonnegative numbers. Let $p \in(0, \infty)$.

(i) If $\sigma_{k} \uparrow$, then

$$
\sup _{k \in \mathbb{Z}}\left(\sum_{m \geq k} a_{m}\right)^{p} \sigma_{k} \approx \sup _{m \in \mathbb{Z}} a_{m}^{p} \sigma_{m} .
$$

(ii) If $\tau_{k} \downarrow$, then

$$
\sup _{k \in \mathbb{Z}}\left(\sum_{m \leq k} a_{m}\right)^{p} \tau_{k} \approx \sup _{m \in \mathbb{Z}} a_{m}^{p} \tau_{m} .
$$

Lemma 3.4. Let $\left\{a_{k}\right\}_{k \in \mathbb{Z}},\left\{\sigma_{k}\right\}_{k \in \mathbb{Z}}$, and $\left\{\tau_{k}\right\}_{k \in \mathbb{Z}}$ be sequences of nonnegative numbers.

(i) If $\sigma_{k} \uparrow$, then

$$
\sup _{k \in \mathbb{Z}}\left(\sup _{m \geq k} a_{m}\right) \sigma_{k} \approx \sup _{m \in \mathbb{Z}} a_{m} \sigma_{m} .
$$

(ii) If $\tau_{k} \downarrow$, then

$$
\sup _{k \in \mathbb{Z}}\left(\sup _{m \leq k} a_{m}\right) \tau_{k} \approx \sup _{m \in \mathbb{Z}} a_{m} \tau_{m} .
$$

Next we shall prove an important lemma on an equivalence of two discretizing sequences.

Lemma 3.5. Let $p, q, r \in(0, \infty)$. Let $u$ be an admissible function. Let $h \in \Omega_{u^{q}}$ and $g \in \Omega_{u^{p}}$. Let $\left\{\mu_{k}\right\}$ be a discretizing sequence of $h$ with respect to $u^{q}$ and let $\left\{\lambda_{k}\right\}$ be a discretizing sequence of $g$ with respect to $u^{p}$. Then

$$
\sum_{k \in \mathbb{Z}} \frac{h\left(\mu_{k}\right)^{\frac{r}{q}}}{g\left(\mu_{k}\right)^{\frac{r}{p}}} \approx \sum_{\ell \in \mathbb{Z}} \frac{h\left(\lambda_{\ell}\right)^{\frac{r}{q}}}{g\left(\lambda_{\ell}\right)^{\frac{r}{p}}}
$$

and

$$
\sup _{t \in(0, \infty)} \frac{h(t)^{\frac{1}{q}}}{g(t)^{\frac{1}{p}}} \approx \sup _{k \in \mathbb{Z}} \frac{h\left(\mu_{k}\right)^{\frac{1}{q}}}{g\left(\mu_{k}\right)^{\frac{1}{p}}} \approx \sup _{\ell \in \mathbb{Z}} \frac{h\left(\lambda_{\ell}\right)^{\frac{1}{q}}}{g\left(\lambda_{\ell}\right)^{\frac{1}{p}}} .
$$


Proof: Since $h\left(\mu_{k}\right) \uparrow$ and $\frac{h\left(\mu_{k}\right)}{u^{q}\left(\mu_{k}\right)} \downarrow$, we have

$$
\sum_{k \in \mathbb{Z}} \frac{h\left(\mu_{k}\right)^{\frac{r}{q}}}{g\left(\mu_{k}\right)^{\frac{r}{p}}}=\sum_{\ell \in \mathbb{Z}} \sum_{\lambda_{\ell} \leq \mu_{k}<\lambda_{\ell+1}} \frac{h\left(\mu_{k}\right)^{\frac{r}{q}}}{g\left(\mu_{k}\right)^{\frac{r}{p}}}=\sum_{\ell \in \mathbb{Z}_{1}}+\sum_{\ell \in \mathbb{Z}_{2}} .
$$

For the first sum, we have by (2.3), (2.7) and (2.8)

$$
\sum_{\ell \in \mathbb{Z}_{1}} \sum_{\lambda_{\ell} \leq \mu_{k}<\lambda_{\ell+1}} \frac{h\left(\mu_{k}\right)^{\frac{r}{q}}}{g\left(\mu_{k}\right)^{\frac{r}{p}}} \lesssim \sum_{\ell \in \mathbb{Z}_{1}} \frac{1}{g\left(\lambda_{\ell+1}\right)^{\frac{r}{p}}} \sum_{\mu_{k}<\lambda_{\ell+1}} h\left(\mu_{k}\right)^{\frac{r}{q}} \lesssim \sum_{\ell \in \mathbb{Z}} \frac{h\left(\lambda_{\ell+1}\right)^{\frac{r}{q}}}{g\left(\lambda_{\ell+1}\right)^{\frac{r}{p}}} .
$$

Similarly,

$$
\sum_{\ell \in \mathbb{Z}_{2}} \sum_{\lambda_{\ell} \leq \mu_{k}<\lambda_{\ell+1}} \frac{h\left(\mu_{k}\right)^{\frac{r}{q}}}{g\left(\mu_{k}\right)^{\frac{r}{p}}} \lesssim \sum_{\ell \in \mathbb{Z}_{2}} \frac{u\left(\lambda_{\ell}\right)^{r}}{g\left(\lambda_{\ell}\right)^{\frac{r}{p}}} \sum_{\lambda_{\ell} \leq \mu_{k}} \frac{h\left(\mu_{k}\right)^{\frac{r}{q}}}{u\left(\mu_{k}\right)^{r}} \lesssim \sum_{\ell \in \mathbb{Z}} \frac{h\left(\lambda_{\ell}\right)^{\frac{r}{q}}}{g\left(\lambda_{\ell}\right)^{\frac{r}{p}}} .
$$

On replacing the roles of $\lambda_{k}$ and $\mu_{k}$, we get the converse inequality.

As for the second assertion, observe that

$$
\sup _{t \in(0, \infty)} \frac{h(t)^{\frac{1}{q}}}{g(t)^{\frac{1}{p}}}=\sup _{k \in \mathbb{Z} \mu_{k} \leq t<\mu_{k+1}} \frac{h(t)^{\frac{1}{q}}}{g(t)^{\frac{1}{p}}} .
$$

If now $k \in \mathbb{Z}_{1}$, then, by (2.3) for $h$ and (2.7) for $g$,

$$
\sup _{\mu_{k} \leq t<\mu_{k+1}} \frac{h(t)^{\frac{1}{q}}}{g(t)^{\frac{1}{p}}} \lesssim \frac{h\left(\mu_{k}\right)^{\frac{1}{q}}}{g\left(\mu_{k}\right)^{\frac{1}{p}}} .
$$

If $k \in \mathbb{Z}_{2}$, then

$$
\sup _{\mu_{k} \leq t<\mu_{k+1}} \frac{h(t)^{\frac{1}{q}}}{g(t)^{\frac{1}{p}}} \lesssim \frac{h\left(\mu_{k}\right)^{\frac{1}{q}}}{u\left(\mu_{k}\right)} \sup _{\mu_{k} \leq t<\mu_{k+1}} \frac{u(t)}{g(t)^{\frac{1}{p}}} \lesssim \frac{h\left(\mu_{k}\right)^{\frac{1}{q}}}{g\left(\mu_{k}\right)^{\frac{1}{p}}} .
$$

We thus conclude that

$$
\sup _{t \in(0, \infty)} \frac{h(t)^{\frac{1}{q}}}{g(t)^{\frac{1}{p}}} \lesssim \sup _{k \in \mathbb{Z}} \frac{h\left(\mu_{k}\right)^{\frac{1}{q}}}{g\left(\mu_{k}\right)^{\frac{1}{p}}}
$$

and by the same way we get

$$
\sup _{t \in(0, \infty)} \frac{h(t)^{\frac{1}{q}}}{g(t)^{\frac{1}{p}}} \lesssim \sup _{\ell \in \mathbb{Z}} \frac{h\left(\lambda_{\ell}\right)^{\frac{1}{q}}}{g\left(\lambda_{\ell}\right)^{\frac{1}{p}}} .
$$

Now we shall prove a discretization lemma for a Hilbert norm, a main result of this section.

We work within the following fixed scheme: $u$ is an admissible function, $\nu$ is a positive measure on $[0, \infty), q \in(0, \infty)$ and

$$
h(t)=\int_{[0, \infty)} \frac{u(t)^{q}}{(u(s)+u(t))^{q}} d \nu(s), \quad t \in(0, \infty) .
$$


Recall that

$$
h(t) \approx \int_{[0, t]} d \nu(s)+u(t)^{q} \int_{[t, \infty)} u(s)^{-q} d \nu(s), \quad t \in(0, \infty) .
$$

We shall further assume that $\nu$ is non-degenerate.

In this setup, we have

Lemma 3.6. Let $q \in(0, \infty)$, let $u$ be an admissible function and let $\nu$ be a non-degenerate positive Borel measure. Let $h$ be the fundamental function of $\nu$ with respect to $u^{q}$ and let $f$ be a measurable function on $[0, \infty)$. Let $\left\{x_{k}\right\}$ be a discretizing sequence for $h$ with respect to $u^{q}$. Then

$$
\begin{aligned}
\int_{0}^{\infty} & \left(\int_{[0, \infty)} \frac{|f(y)|}{u(x)+u(y)} d y\right)^{q} d \nu(x) \\
& \approx \sum_{k \in \mathbb{Z}}\left(\int_{[0, \infty)} \frac{|f(y)|}{u\left(x_{k}\right)+u(y)} d y\right)^{q} h\left(x_{k}\right) \\
\approx & \sum_{k \in \mathbb{Z}}\left(u^{-1}\left(x_{k}\right) \int_{x_{k-1}}^{x_{k}}|f(y)| d y+\int_{x_{k}}^{x_{k+1}}|f(y)| u(y)^{-1} d y\right)^{q} h\left(x_{k}\right) \\
\approx & \sum_{k \in \mathbb{Z}_{1}}\left(\int_{x_{k}}^{x_{k+1}}|f(y)| u(y)^{-1} d y\right)^{q} h\left(x_{k}\right) \\
& +\sum_{k \in \mathbb{Z}_{2}}\left(\int_{x_{k}}^{x_{k+1}}|f(y)| d y\right)^{q} u\left(x_{k}\right)^{-q} h\left(x_{k}\right) \\
\approx & \sum_{k \in \mathbb{Z}}\left(\int_{x_{k}}^{x_{k+1}}|f(y)| u(y)^{-1} h(y)^{\frac{1}{q}} d y\right)^{q} .
\end{aligned}
$$

Proof: Clearly, the function $g(x)=u(x) \int_{0}^{\infty} \frac{|f(y)|}{u(x)+u(y)} d y$ belongs to $\Omega_{u}$. Using Corollary 2.13 with the measure $u(x)^{-q} d \nu$, we obtain the first relation. 
Since

$$
\int_{0}^{\infty} \frac{|f(y)|}{u\left(x_{k}\right)+u(y)} d y \approx u\left(x_{k}\right)^{-1} \int_{0}^{x_{k}}|f(y)| d y+\int_{x_{k}}^{\infty}|f(y)| u(y)^{-1} d y
$$

$h\left(x_{k}\right) \uparrow$ and $\frac{h\left(x_{k}\right)}{u\left(x_{k}\right)^{q}} \downarrow$, we get the second relation by Lemma 3.1. The converse inequality is trivial.

To prove the third equivalence, it suffices to show that

$$
\begin{aligned}
& \sum_{k \in \mathbb{Z}}\left(u^{-1}\left(x_{k}\right) \int_{x_{k-1}}^{x_{k}}|f(y)| d y+\int_{x_{k}}^{x_{k+1}}|f(y)| u(y)^{-1} d y\right)^{q} h\left(x_{k}\right) \\
& \lesssim \sum_{k \in \mathbb{Z}_{1}}\left(\int_{x_{k}}^{x_{k+1}}|f(y)| u(y)^{-1} d y\right)^{q} h\left(x_{k}\right) \\
& \quad+\sum_{k \in \mathbb{Z}_{2}}\left(\int_{x_{k}}^{x_{k+1}}|f(y)| d y\right)^{q} u\left(x_{k}\right)^{-q} h\left(x_{k}\right) .
\end{aligned}
$$

When $k-1 \in \mathbb{Z}_{1}$, we have $h\left(x_{k}\right)=a h\left(x_{k-1}\right)$. Thus,

$$
\begin{aligned}
\sum_{k \in \mathbb{Z}} u^{-q}\left(x_{k}\right) & \left(\int_{x_{k-1}}^{x_{k}}|f(y)| d y\right)^{q} h\left(x_{k}\right) \\
\leq & a \sum_{k-1 \in \mathbb{Z}_{1}}\left(\int_{x_{k-1}}^{x_{k}}|f(y)| u(y)^{-1} d y\right)^{q} h\left(x_{k-1}\right) \\
& +\sum_{k-1 \in \mathbb{Z}_{2}}\left(\int_{x_{k-1}}^{x_{k}}|f(y)| d y\right)^{q} u\left(x_{k-1}\right)^{-q} h\left(x_{k-1}\right) \\
= & a \sum_{k \in \mathbb{Z}_{1}}\left(\int_{x_{k}}^{x_{k+1}}|f(y)| u(y)^{-1} d y\right)^{q} h\left(x_{k}\right) \\
& +\sum_{k \in \mathbb{Z}_{2}}\left(\int_{x_{k}}^{x_{k+1}}|f(y)| d y\right)^{q} u\left(x_{k}\right)^{-q} h\left(x_{k}\right) .
\end{aligned}
$$


Using the monotonicity of $u$, we obtain the remaining estimate

$$
\begin{aligned}
\sum_{k \in \mathbb{Z}} & \left(\int_{x_{k}}^{x_{k+1}}|f(y)| u(y)^{-1} d y\right)^{q} h\left(x_{k}\right) \\
& \lesssim \sum_{k \in \mathbb{Z}_{1}}\left(\int_{x_{k}}^{x_{k+1}}|f(y)| u(y)^{-1} d y\right)^{q} h\left(x_{k}\right) \\
& +\sum_{k \in \mathbb{Z}_{2}}\left(\int_{x_{k}}^{x_{k+1}}|f(y)| d y\right)^{q} u\left(x_{k}\right)^{-q} h\left(x_{k}\right) .
\end{aligned}
$$

Finally, the last equivalence is a simple consequence of (2.3) and (2.4).

Lemma 3.7. Let $q \in(0, \infty)$, let $u$ be an admissible function and let $\nu$ be a non-degenerate positive Borel measure. Let $h$ be the fundamental function of $\nu$ with respect to $u^{q}$ and let $f$ be a measurable function on $[0, \infty)$. Let $\left\{x_{k}\right\}$ be a discretizing sequence for $h$ with respect to $u^{q}$. Then

$$
\begin{aligned}
& \left(\int_{[0, \infty)}\left(\sup _{y \in(0, \infty)} \frac{|f(y)|}{u(x)+u(y)}\right)^{q} d \nu(x)\right) \\
& \approx \sum_{k \in \mathbb{Z}}\left(\sup _{y \in(0, \infty)} \frac{|f(y)|}{u\left(x_{k}\right)+u(y)}\right)^{q} h\left(x_{k}\right) \\
& \approx \sum_{k \in \mathbb{Z}}\left(u^{-1}\left(x_{k}\right) \sup _{x_{k-1} \leq y<x_{k}}|f(y)|+\sup _{x_{k} \leq y<x_{k+1}}|f(y)| u(y)^{-1}\right)^{q} h\left(x_{k}\right) \\
& \approx \sum_{k \in \mathbb{Z}_{1}} \sup _{x_{k} \leq y<x_{k+1}}|f(y)|^{q} u(y)^{-q} h\left(x_{k}\right) \\
& +\sum_{k \in \mathbb{Z}_{2}} \sup _{x_{k} \leq y<x_{k+1}}|f(y)|^{q} u\left(x_{k}\right)^{-q} h\left(x_{k}\right) \\
& \approx \sum_{k \in \mathbb{Z}} \sup _{x_{k} \leq y<x_{k+1}}|f(y)|^{q} u(y)^{-q} h(y) .
\end{aligned}
$$

Proof: This follows on using Corollary 2.13 and Lemma 3.2. 
Lemma 3.8. Let $q \in(0, \infty)$. Let $u$ be an admissible function and let $\varphi \in \Omega_{u^{q}}$. Let $\left\{x_{k}\right\}$ be a discretizing sequence for $\varphi$ with respect to $u^{q}$. Let $f$ be a measurable function on $[0, \infty)$. Then

$$
\begin{aligned}
& \sup _{x \in(0, \infty)} \varphi(x)\left(\int_{0}^{\infty} \frac{|f(y)|}{u(x)+u(y)} d y\right)^{q} \\
\approx & \sup _{k \in \mathbb{Z}} \varphi\left(x_{k}\right)\left(\int_{0}^{\infty} \frac{|f(y)|}{u\left(x_{k}\right)+u(y)} d y\right)^{q} \\
\approx & \sup _{k \in \mathbb{Z}} \varphi\left(x_{k}\right) u\left(x_{k}\right)^{-q}\left(\int_{x_{k-1}}^{x_{k}}|f(y)| d y\right)^{q} \\
+ & \sup _{k \in \mathbb{Z}} \varphi\left(x_{k}\right)\left(\int_{x_{k}}^{x_{k+1}}|f(y)| u(y)^{-1} d y\right)^{q} \\
\approx & \sup _{k \in \mathbb{Z}} \varphi\left(x_{k}\right)\left(\int_{x_{k}}^{x_{k+1}}|f(y)| u(y)^{-1} d y\right)^{q} \\
& +\sup _{k \in \mathbb{Z}_{2}} \varphi\left(x_{k}\right) u\left(x_{k}\right)^{-q}\left(\int_{x_{k}}^{x_{k+1}}|f(y)| d y\right)^{q} \\
\approx & \sup _{k \in \mathbb{Z}}\left(\int_{x_{k}}^{x_{k+1}}|f(y)| u(y)^{-1} \varphi(y)^{\frac{1}{q}} d y\right)^{q} .
\end{aligned}
$$

Proof: This follows from using Lemmas 3.3 and 3.5.

Lemma 3.9. Let $u$ be an admissible function and let $\varphi \in \Omega_{u}$. Let $\left\{x_{k}\right\}$ be a discretizing sequence for $\varphi$ with respect to $u$. Then

$$
\begin{aligned}
& \sup _{x \in(0, \infty)} \varphi(x) \sup _{0<y<\infty} \frac{|f(y)|}{u(x)+u(y)} \\
\approx & \sup _{k \in \mathbb{Z}} \varphi\left(x_{k}\right) \sup _{0<y<\infty} \frac{|f(y)|}{u\left(x_{k}\right)+u(y)} \\
\approx & \sup _{k \in \mathbb{Z}} \varphi\left(x_{k}\right) u\left(x_{k}\right)^{-1} \sup _{x_{k-1}<y<x_{k}}|f(y)| \\
& +\sup _{k \in \mathbb{Z}} \varphi\left(x_{k}\right) \sup _{x_{k}<y<x_{k+1}}|f(y)| u(y)^{-1} \\
\approx & \sup _{k \in \mathbb{Z}_{1}} \varphi\left(x_{k}\right) \sup _{x_{k} \leq y<x_{k+1}}|f(y)| u(y)^{-1} \\
& +\sup _{k \in \mathbb{Z}_{2}} \varphi\left(x_{k}\right) u\left(x_{k}\right)^{-1} \sup _{x_{k}<y<x_{k+1}}|f(y)| \\
\approx & \sup _{k \in \mathbb{Z} x_{k} \leq y<x_{k+1}} \sup _{|f(y)| u(y)^{-1} \varphi(y) .}
\end{aligned}
$$

Proof: This follows from using Lemmas 3.4 and 3.5. 


\section{Embeddings of classical Lorentz spaces, type $\Gamma \hookrightarrow \Lambda$}

As our first main application we shall establish necessary and sufficient conditions such that the inequality

$$
\left(\int_{0}^{\infty}\left(f^{*}(t)\right)^{q} w(t) d t\right)^{\frac{1}{q}} \leq C\left(\int_{0}^{\infty}\left(f_{u}^{* *}(t)\right)^{p} v(t) d t\right)^{\frac{1}{p}}
$$

holds for every $f \in \mathcal{M}(\mathcal{R}, \mu)$, where $f_{u}^{* *}(t)=\frac{1}{U(t)} \int_{0}^{t} f^{*}(s) u(s) d s, u, v, w$ are weights, $U(t)=\int_{0}^{t} u(s) d s$, and $p, q \in(0, \infty)$.

In the proof of the necessity part of our main theorem we will need the following version of the classical Landau resonance theorem.

Proposition 4.1. Let $\left\{w_{k}\right\}$ and $\left\{v_{k}\right\}, k \in \mathbb{Z}$, be two (double-infinite) sequences of positive real numbers. Let $p, q \in(0, \infty)$ and assume that the inequality

$$
\left(\sum_{k \in \mathbb{Z}} a_{k}^{q} v_{k}\right)^{\frac{1}{q}} \lesssim\left(\sum_{k \in \mathbb{Z}} a_{k}^{p} w_{k}\right)^{\frac{1}{p}}
$$

is satisfied for every sequence $\left\{a_{k}\right\}$ of non-negative real numbers.

(i) If $p \leq q$, then

$$
\sup _{k \in \mathbb{Z}} w_{k}^{-\frac{q}{p}} v_{k}<\infty
$$

(ii) If $p>q$, then

$$
\left(\sum_{k \in \mathbb{Z}} w_{k}^{-\frac{r}{p}} v_{k}^{\frac{r}{q}}\right)^{\frac{1}{r}}<\infty, \quad \text { where } r=\frac{p q}{p-q} .
$$

This assertion is well known. For the sake of completeness, let us just point out that a simple direct proof of (i) is seen from setting, for a given $k \in \mathbb{Z}$,

$$
a_{j}= \begin{cases}w_{k}^{-\frac{1}{p}} & \text { when } j=k \\ 0 & \text { when } j \neq k\end{cases}
$$

while (ii) follows on putting

$$
a_{k}= \begin{cases}\left(\frac{v_{k}}{w_{k}}\right)^{\frac{1}{p-q}} & \text { when } k \in[-N, N] \\ 0 & \text { otherwise. }\end{cases}
$$


This shows

$$
\left(\sum_{k=-N}^{N} w_{k}^{-\frac{r}{p}} v_{k}^{\frac{r}{q}}\right)^{\frac{1}{r}} \leq C
$$

with $C$ independent of $N$, and we just have to let $N \rightarrow \infty$.

Our main result reads as follows.

Theorem 4.2. Let $u, v, w$ be locally integrable weights on $[0, \infty)$. Let $p, q \in(0, \infty)$. When $p>q$, we set $r=\frac{p q}{p-q}$. Assume that $u$ is such that $U^{p}$ is admissible and the measure $v(t) d t$ is non-degenerate with respect to $U^{p}$.

(i) If $0<p \leq q<\infty$ and $1 \leq q<\infty$, then (4.1) holds for some $C>0$ and all $f$ if and only if

$$
A(1)=\sup _{t \in(0, \infty)} \frac{W(t)^{\frac{1}{q}}}{\left(V(t)+U(t)^{p} \int_{t}^{\infty} U(s)^{-p} v(s) d s\right)^{\frac{1}{p}}}<\infty .
$$

(ii) If $1 \leq q<p<\infty$, then (4.1) holds for some $C>0$ and all $f$ if and only if

$$
\begin{aligned}
& A(2)=\left(\int_{0}^{\infty} \frac{U(t)^{r}\left[\sup _{y \in[t, \infty)} U(y)^{-r} W(y)^{\frac{r}{q}}\right]}{\left(V(t)+U(t)^{p} \int_{t}^{\infty} U(s)^{-p} v(s) d s\right)^{\frac{r}{p}+2}}\right. \\
& \left.\times V(t) \int_{t}^{\infty} U(s)^{-p} v(s) d s d\left(U^{p}(t)\right)\right)^{\frac{1}{r}}<\infty .
\end{aligned}
$$

(iii) If $0<p \leq q<1$, then (4.1) holds for some $C>0$ and all $f$ if and only if

$$
A(3)=\sup _{t \in(0, \infty)} \frac{W(t)^{\frac{1}{q}}+U(t)\left(\int_{t}^{\infty} W(s)^{\frac{q}{1-q}} w(s) U(s)^{-\frac{q}{1-q}} d s\right)^{\frac{1-q}{q}}}{\left(V(t)+U(t)^{p} \int_{t}^{\infty} U(s)^{-p} v(s) d s\right)^{\frac{1}{p}}}<\infty
$$


(iv) If $0<q<1$ and $0<q<p$, then (4.1) holds for some $C>0$ and all $f$ if and only if $A(4)<\infty$, where

$$
\begin{aligned}
& A(4)=\left(\int_{0}^{\infty} \frac{\left[W(t)^{\frac{1}{1-q}}+U(t)^{\frac{q}{1-q}} \int_{t}^{\infty} W(s)^{\frac{q}{1-q}} w(s) U(s)^{-\frac{q}{1-q}} d s\right]^{\frac{r(1-q)}{q}-1}}{\left(V(t)+U(t)^{p} \int_{t}^{\infty} U(s)^{-p} v(s) d s\right)^{\frac{r}{p}}}\right. \\
& \left.\times W(t)^{\frac{q}{1-q}} w(t) d t\right)^{\frac{1}{r}}
\end{aligned}
$$

Moreover, $A(4) \approx A(5)$, where

$$
\begin{array}{r}
A(5)=\left(\int_{0}^{\infty} \frac{\left[W(t)^{\frac{1}{1-q}}+U(t)^{\frac{q}{1-q}} \int_{t}^{\infty} W(s)^{\frac{q}{1-q}} w(s) U(s)^{-\frac{q}{1-q}} d s\right]^{\frac{r(1-q)}{q}}}{\left(V(t)+U(t)^{p} \int_{t}^{\infty} U(s)^{-p} v(s) d s\right)^{\frac{r}{p}+2}}\right. \\
\left.\times V(t) \int_{t}^{\infty} U(s)^{-p} v(s) d s d\left(U^{p}(t)\right)\right)^{\frac{1}{r}} .
\end{array}
$$

Proof: We start with the upper bounds (sufficiency). First, a standard argument shows that it is enough to prove (4.1) for $f$ satisfying $f^{*}(t)=$ $\int_{t}^{\infty} h(s) d s$, where $h$ is some positive measurable function on $(0, \infty)$. That is, we only have to prove

$$
\begin{aligned}
\left(\int _ { 0 } ^ { \infty } \left(\int_{t}^{\infty} h(s)\right.\right. & \left.d s)^{q} w(t) d t\right)^{\frac{1}{q}} \\
& \leq C\left(\int_{0}^{\infty}\left(\int_{0}^{\infty} \frac{U(s) h(s)}{U(s)+U(t)} d s\right)^{p} v(t) d t\right)^{\frac{1}{p}}
\end{aligned}
$$

for every $h \geq 0$.

Define

$$
\varphi(t)=U(t)^{p} \int_{0}^{\infty} \frac{v(s)}{(U(s)+U(t))^{p}} d s .
$$

Then $\varphi \in \Omega_{U^{p}}$, and therefore there exists a discretizing sequence for $\varphi$ with respect to $U^{p}$. Let $\left\{x_{k}\right\}$ be one such sequence. Then $\varphi\left(x_{k}\right) \uparrow \uparrow$ and $\frac{\varphi\left(x_{k}\right)}{U\left(x_{k}\right)^{p}} \downarrow$. Furthermore, there is a decomposition $\mathbb{Z}=\mathbb{Z}_{1} \cup \mathbb{Z}_{2}, \mathbb{Z}_{1} \cap \mathbb{Z}_{2}=\emptyset$ such that for every $k \in \mathbb{Z}_{1}$ and $t \in \Delta_{k}=\left[x_{k}, x_{k+1}\right], \varphi(t) \approx \varphi\left(x_{k}\right)$ and 
for every $k \in \mathbb{Z}_{2}$ and $t \in \Delta_{k}, \frac{\varphi(t)}{U(t)^{p}} \approx \frac{\varphi\left(x_{k}\right)}{U\left(x_{k}\right)^{p}}$. By Lemma 3.6, applied to $f=h U$ and $h=\varphi$, we have

$$
\begin{aligned}
& \left(\int_{0}^{\infty}\left(\int_{0}^{\infty} \frac{U(s) h(s)}{U(s)+U(t)} d s\right)^{p} v(t) d t\right)^{\frac{1}{p}} \\
& \quad \approx\left(\sum_{k \in \mathbb{Z}}\left(\int_{0}^{\infty} \frac{U(s) h(s)}{U\left(x_{k}\right)+U(s)} d s\right)^{p} \varphi\left(x_{k}\right)\right)^{\frac{1}{p}} \\
& \approx\left(\sum_{k \in \mathbb{Z}}\left(\int_{x_{k}}^{x_{k+1}} h(s) \varphi(s)^{\frac{1}{p}} d s\right)^{p}\right)^{\frac{1}{p}} .
\end{aligned}
$$

For the left side of (4.2), we get

$$
\begin{aligned}
& \int_{0}^{\infty}\left(\int_{t}^{\infty} h(s) d s\right)^{q} w(t) d t \\
& =\sum_{k \in \mathbb{Z}} \int_{x_{k}}^{x_{k+1}}\left(\int_{t}^{\infty} h(s) d s\right)^{q} w(t) d t \\
& \approx \sum_{k \in \mathbb{Z}} \int_{x_{k}}^{x_{k+1}}\left(\int_{t}^{x_{k+1}} h(s) d s\right)^{q} w(t) d t \\
& \quad+\sum_{k \in \mathbb{Z}} \int_{x_{k}}^{x_{k+1}} w(t) d t\left(\int_{x_{k+1}}^{\infty} h(s) d s\right)^{q}=\mathrm{I}+\mathrm{II} .
\end{aligned}
$$

Now we shall distinguish several cases. Assume first that $1 \leq q<\infty$. We shall use the Hardy inequality (cf. [24])

$$
\begin{aligned}
\int_{x_{k}}^{x_{k+1}}\left(\int_{t}^{x_{k+1}} h(s) d s\right)^{q} w(t) d t & \\
& \lesssim\left(\int_{x_{k}}^{x_{k+1}} h(s) \varphi(s)^{\frac{1}{p}} d s\right)^{q} \sup _{t \in\left[x_{k}, x_{k+1}\right]} \varphi(t)^{-\frac{q}{p}} \int_{x_{k}}^{t} w(s) d s,
\end{aligned}
$$

where the constant does not depend on $\left\{x_{k}\right\}$. We obtain

$$
\mathrm{I} \lesssim \sum_{k \in \mathbb{Z}}\left(\int_{x_{k}}^{x_{k+1}} h(s) \varphi(s)^{\frac{1}{p}} d s\right)^{q} \sup _{t \in\left[x_{k}, x_{k+1}\right]} \varphi(t)^{-\frac{q}{p}} \int_{x_{k}}^{t} w(y) d y .
$$


Let $p \leq q$, Using (4.4) and Lemma 3.9,

$$
\begin{aligned}
\mathrm{I} \lesssim & \sum_{k \in \mathbb{Z}}\left(\int_{x_{k}}^{x_{k+1}} h(s) \varphi(s)^{\frac{1}{p}} d s\right)^{q} \\
& \times \sup _{k \in \mathbb{Z}} \sup _{t \in\left[x_{k}, x_{k+1}\right]} \varphi(t)^{-\frac{q}{p}} \int_{0}^{t} w(y) d y \\
\lesssim & \left(\sum_{k \in \mathbb{Z}}\left(\int_{x_{k}}^{x_{k+1}} h(s) \varphi(s)^{\frac{1}{p}} d s\right)^{p}\right)^{\frac{q}{p}} \\
& \times \sup _{t \in(0, \infty)} \varphi(t)^{-\frac{q}{p}} \int_{0}^{t} w(y) d y \\
\lesssim & \left(\int_{0}^{\infty}\left(\int_{0}^{\infty} \frac{U(s) h(s)}{U(s)+U(t)} d s\right)^{p} v(t) d t\right)^{\frac{q}{p}} \\
& \quad \times \sup _{t \in(0, \infty)} \varphi(t)^{-\frac{q}{p}} \int_{0}^{t} w(y) d y \\
& A(1)^{q}\left(\int_{0}^{\infty}\left(\int_{0}^{\infty} \frac{U(s) h(s)}{U(s)+U(t)} d s\right)^{p} v(t) d t\right)^{\frac{q}{p}} .
\end{aligned}
$$

Finally, we also have

$$
\begin{aligned}
\mathrm{II} & \leq\left(\sum_{k \in \mathbb{Z}}\left(\int_{x_{k+1}}^{\infty} h(s) d s\right)^{p} \varphi\left(x_{k+1}\right)\right)^{\frac{q}{p}} \sup _{k \in \mathbb{Z}} \varphi\left(x_{k+1}\right)^{-\frac{q}{p}} \int_{0}^{x_{k+1}} w(t) d t \\
& \leq A(1)^{q}\left(\int_{0}^{\infty}\left(\int_{0}^{\infty} \frac{U(s) h(s)}{U(s)+U(t)} d s\right)^{p} v(t) d t\right)^{\frac{q}{p}},
\end{aligned}
$$

and (4.2) follows from (4.5), (4.7) and (4.8). 
Now assume that $1 \leq q<p$. Using the Hölder inequality for sums with exponents $\frac{p}{q}$ and $\frac{p}{p-q}$ in (4.6), we get

$$
\begin{aligned}
\mathrm{I} \lesssim\left(\sum _ { k \in \mathbb { Z } } \left(\int_{x_{k}}^{x_{k+1}} h(s)\right.\right. & \left.\left.\varphi(s)^{\frac{1}{p}} d s\right)^{p}\right)^{\frac{q}{p}} \\
& \times\left(\sum_{k \in \mathbb{Z}} \sup _{t \in\left[x_{k}, x_{k+1}\right]} \varphi(t)^{-\frac{r}{p}}\left(\int_{x_{k}}^{t} w(y) d y\right)^{\frac{r}{q}}\right)^{\frac{q}{r}} .
\end{aligned}
$$

Now on employing (4.4), Lemma 3.7 and Theorem 2.15 (applied to parameters $g=\varphi, x_{k}=\mu_{k}, d \nu(t)=v(t) d t, u^{q}=U^{p}$ ), we obtain

$$
\begin{aligned}
\mathrm{I} \leq & \left(\int_{0}^{\infty}\left(\int_{0}^{\infty} \frac{U(s) h(s)}{U(s)+U(t)} d s\right)^{p} v(t) d t\right)^{\frac{q}{p}} \\
& \times\left(\int_{0}^{\infty} \frac{U(t)^{r}\left[\sup _{s \in[t, \infty)} U(s)^{-r} W(s)^{\frac{r}{q}}\right]}{\varphi(t)^{\frac{r}{p}+2}}\right. \\
& \left.\times V(t) \int_{t}^{\infty} U(y)^{-p} v(y) d y\right)^{\frac{q}{p}} d\left(U^{p}\right)(t) \\
\leq & A(2)^{q}\left(\int_{0}^{\infty}\left(\int_{0}^{\infty} \frac{U(s) h(s)}{U(s)+U(t)} d s\right)^{p} v(t) d t\right)^{\frac{q}{p}} .
\end{aligned}
$$

Similarly we obtain

$\mathrm{II} \lesssim\left(\sum_{k \in \mathbb{Z}}\left(\int_{x_{k+1}}^{x_{k+2}} h(s) d s\right)^{p} \varphi\left(x_{k+1}\right)\right)^{\frac{q}{p}}\left(\sum_{k \in \mathbb{Z}} W\left(x_{k+1}\right)^{\frac{r}{q}} \varphi\left(x_{k+1}\right)^{-\frac{r}{p}}\right)^{\frac{q}{r}}$.

On using (4.4), we get

$$
\begin{aligned}
\mathrm{II} \leq\left(\int_{0}^{\infty}\left(\int_{0}^{\infty} \frac{U(s) h(s)}{U(s)+U(t)} d s\right)^{p} v(t) d t\right)^{\frac{q}{p}} & \\
& \times\left(\sum_{k \in \mathbb{Z}} W\left(x_{k}\right)^{\frac{r}{q}} \varphi\left(x_{k}\right)^{-\frac{r}{p}}\right)^{\frac{q}{r}} .
\end{aligned}
$$


As

$$
W\left(x_{k}\right)^{\frac{r}{q}} \leq\left(U\left(x_{k}\right)^{q} \sup _{t \in\left[x_{k}, \infty\right)} U(t)^{-q} W(t)\right)^{\frac{r}{q}},
$$

we obtain from Theorem 2.15

$$
\mathrm{II} \leq A(2)^{q}\left(\int_{0}^{\infty}\left(\int_{0}^{\infty} \frac{U(s) h(s)}{U(s)+U(t)} d s\right)^{p} v(t) d t\right)^{\frac{q}{p}} .
$$

From (4.5), (4.9) and (4.11) we get (4.2).

Now let us assume that $0<q<1$. By a simple modification of $[\mathbf{2 8}$, Theorem 3.3], we have

$$
\begin{aligned}
& \int_{x_{k}}^{x_{k+1}}\left(\int_{t}^{x_{k+1}} h(s) d s\right)^{q} w(t) d t \\
\lesssim & \left(\int_{x_{k}}^{x_{k+1}} h(s) \varphi(s)^{\frac{1}{p}} d s\right)^{q}\left(\int_{x_{k}}^{x_{k+1}}\left(\int_{x_{k}}^{t} w(y) d y\right)^{\frac{q}{1-q}} w(t) \varphi(t)^{-\frac{q}{p(1-q)}} d t\right)^{1-q}
\end{aligned}
$$

with constant independent of $x_{k}$. We get

$$
\begin{aligned}
\mathrm{I} \lesssim \sum_{k \in \mathbb{Z}} & \left(\int_{x_{k}}^{x_{k+1}} h(s) \varphi(s)^{\frac{1}{p}} d s\right)^{q} \\
& \times\left(\int_{x_{k}}^{x_{k+1}}\left(\int_{x_{k}}^{t} w(y) d y\right)^{\frac{q}{1-q}} w(t) \varphi(t)^{-\frac{q}{p(1-q)}} d t\right)^{1-q} .
\end{aligned}
$$

Let $0<p \leq q<1$, then, by (4.4) and Lemma 3.8,

$$
\begin{aligned}
\mathrm{I} \lesssim & \sum_{k \in \mathbb{Z}}\left(\int_{x_{k}}^{x_{k+1}} h(s) \varphi(s)^{\frac{1}{p}} d s\right)^{q} \\
& \times \sup _{k \in \mathbb{Z}}\left(\int_{x_{k}}^{x_{k+1}} W(t)^{\frac{q}{1-q}} w(t) \varphi(t)^{-\frac{q}{p(1-q)}} d t\right)^{1-q} \\
\lesssim & \left(\int_{0}^{\infty}\left(\int_{0}^{\infty} \frac{U(s) h(s)}{U(s)+U(t)} d s\right)^{p} v(t) d t\right)^{\frac{q}{p}} \\
& \times \sup _{t \in(0, \infty)} \frac{W^{\infty}(t)+U(t)^{q}\left(\int_{t}^{\infty} W(y)^{\frac{q}{1-q}} w(y) U(y)^{-\frac{q}{1-q}} d y\right)^{1-q}}{\varphi(t)^{\frac{q}{p}}} .
\end{aligned}
$$

As $A(1) \leq A(3)$, we can combine (4.5), (4.13), and (4.8) to obtain (4.2). 
Let now $0<q<1$ and $q<p$. Using the Hölder inequality in (4.12) with exponents $\frac{p}{q}$ and $\frac{r}{q}$ and then (4.4) and Lemma 3.6, we get

$$
\begin{aligned}
\mathrm{I} \lesssim & \left(\sum_{k \in \mathbb{Z}}\left(\int_{x_{k}}^{x_{k+1}} h(s) \varphi(s)^{\frac{1}{p}} d s\right)^{p}\right)^{\frac{q}{p}} \\
& \times\left(\sum_{k \in \mathbb{Z}}\left(\int_{x_{k}}^{x_{k+1}} W(t)^{\frac{q}{1-q}} w(t) \varphi(t)^{-\frac{q}{p(1-q)}} d t\right)^{\frac{r(1-q)}{q}}\right)^{\frac{q}{r}} \\
\lesssim & \left(\int_{0}^{\infty}\left(\int_{0}^{\infty} \frac{U(s) h(s)}{U(s)+U(t)} d s\right)^{p} v(t) d t\right)^{\frac{q}{p}} \\
& \times\left(\sum_{k \in \mathbb{Z}} \frac{W\left(x_{k}\right)^{\frac{r}{q}}+\left(U\left(x_{k}\right)^{\frac{q}{1-q}} \int_{x_{k}}^{\infty} W(t)^{\frac{q}{1-q}} w(t) U(t)^{-\frac{q}{1-q}} d t\right)^{\frac{r(1-q)}{q}}}{\varphi\left(x_{k}\right)^{\frac{r}{p}}}\right) .
\end{aligned}
$$

Now, combining (4.14) and (4.10) (note that (4.10) was in fact established for every $q \in(0, \infty))$, we have

$$
\begin{gathered}
\mathrm{I}+\mathrm{II} \leq\left(\int_{0}^{\infty}\left(\int_{0}^{\infty} \frac{U(s) h(s)}{U(s)+U(t)} d s\right)^{p} v(t) d t\right)^{\frac{q}{p}} \\
\times\left(\sum_{k \in \mathbb{Z}} \frac{W\left(x_{k}\right)^{\frac{r}{q}}+\left(U\left(x_{k}\right)^{\frac{q}{1-q}} \int_{x_{k}}^{\infty} W(t)^{\frac{q}{1-q}} w(t) U(t)^{-\frac{q}{1-q}} d t\right)^{\frac{r(1-q)}{q}}}{\varphi\left(x_{k}\right)^{\frac{r}{p}}}\right)^{\frac{q}{r}} .
\end{gathered}
$$

Applying Theorem 2.11 to the function

$$
\int_{0}^{x} W(t)^{\frac{q}{1-q}} w(t) d t+U(x)^{\frac{q}{1-q}} \int_{x}^{\infty} W(t)^{\frac{q}{1-q}} w(t) U(t)^{-\frac{q}{1-q}} d t
$$

in place of $h$ and $\varphi$ in place of $\sigma$, we get that the last term is bounded above by $A(4)$. Hence, we get (4.2). Using Theorem 2.14, we see that $A(4) \approx A(5)$. 
Necessity: Let $\left\{x_{k}\right\}$ be a discretizing sequence for $\varphi$ from (4.3). By (4.4) and (4.1),

$$
\begin{aligned}
\left(\sum_{k \in \mathbb{Z}} \int_{x_{k}}^{x_{k+1}}\left(\int_{t}^{x_{k+1}} h(s) d s\right)^{q} w(t) d t\right)^{\frac{1}{q}} & \\
& \lesssim\left(\sum_{k \in \mathbb{Z}}\left(\int_{x_{k}}^{x_{k+1}} h(s) \varphi(s)^{\frac{1}{p}} d s\right)^{p}\right)^{\frac{1}{p}}
\end{aligned}
$$

Let $1 \leq q<\infty$. For $k \in \mathbb{Z}$, let $h_{k}$ be functions that saturate the Hardy inequality (cf. [24]), that is, functions satisfying $\operatorname{supp} h_{k} \subset\left[x_{k}, x_{k+1}\right.$ ), $\int_{x_{k}}^{x^{k+1}} \varphi(s)^{\frac{1}{p}} h_{k}(s) d s=1$, and

$$
\int_{x_{k}}^{x_{k+1}}\left(\int_{t}^{x_{k+1}} h_{k}(s) d s\right)^{q} w(t) d t \gtrsim \sup _{x_{k}<t \leq x_{k+1}} \varphi(t)^{-\frac{q}{p}} \int_{x_{k}}^{t} w(y) d y .
$$

Then we define the test function

$$
h(s)=\sum a_{k} h_{k}(s)
$$

where $\left\{a_{k}\right\}$ is a sequence of non-negative real numbers.

We thus have

$$
\left(\sum_{k \in \mathbb{Z}} a_{k}^{q} \sup _{x_{k}<t \leq x_{k+1}} \varphi(t)^{-\frac{q}{p}} \int_{x_{k}}^{t} w(s) d s\right)^{\frac{1}{q}} \lesssim\left(\sum_{k \in \mathbb{Z}} a_{k}^{p}\right)^{\frac{1}{p}}
$$

Let $p \leq q$. Then, by Proposition 4.1,

$$
\sup _{k \in \mathbb{Z}} \sup _{t \in\left(x_{k}, x_{k+1}\right]} \varphi(t)^{-\frac{q}{p}} \int_{x_{k}}^{t} w(s) d s<\infty .
$$


By Lemma 3.3 (ii),

$$
\begin{aligned}
A(1) & \lesssim \sup _{k \in \mathbb{Z}} \sup _{t \in\left(x_{k}, x_{k+1}\right]} \varphi(t)^{-\frac{1}{p}} W(t)^{\frac{1}{q}} \\
& \lesssim \sup _{k \in \mathbb{Z}} \sup _{t \in\left(x_{k}, x_{k+1}\right]} \varphi(t)^{-\frac{1}{p}}\left(\int_{x_{k}}^{t} w(s) d s\right)^{\frac{1}{q}}+\sup _{k \in \mathbb{Z}} \varphi\left(x_{k}\right)^{-\frac{1}{p}} W\left(x_{k}\right)^{\frac{1}{q}} \\
& \lesssim \sup _{k \in \mathbb{Z}} \sup _{t \in\left(x_{k}, x_{k+1}\right]} \varphi(t)^{-\frac{1}{p}}\left(\int_{x_{k}}^{t} w(s) d s\right)^{\frac{1}{q}}+\sup _{k \in \mathbb{Z}} \varphi\left(x_{k}\right)^{-\frac{1}{p}}\left(\int_{x_{k-1}}^{x_{k}} w(s) d s\right)^{\frac{1}{q}} \\
& \lesssim \sup _{k \in \mathbb{Z}} \sup _{t \in\left(x_{k}, x_{k+1}\right]} \varphi(t)^{-\frac{1}{p}}\left(\int_{x_{k}}^{t} w(s) d s\right)^{\frac{1}{q}}<\infty .
\end{aligned}
$$

Let $q<p$. We obtain from (4.16) and Proposition 4.1

$$
\left(\sum_{k \in \mathbb{Z}}\left(\sup _{x_{k}<t \leq x_{k+1}} \varphi(t)^{-\frac{q}{p}} \int_{x_{k}}^{t} w(s) d s\right)^{\frac{r}{q}}\right)^{\frac{1}{r}}<\infty .
$$

By Theorem 2.15 (applied to $\mu_{k}=x_{k}, g=\varphi, u^{q}=U^{p}$ and $d \nu(t)=$ $v(t) d t$ ), Lemma 3.2 and Lemma 3.1, we have by Lemma 3.7

$$
\begin{aligned}
A(2) & \lesssim\left(\sum_{k \in \mathbb{Z}} \frac{U\left(x_{k}\right)^{r} \sup _{s \in\left[x_{k}, \infty\right)} U(s)^{-r} W(s)^{\frac{r}{q}}}{\varphi\left(x_{k}\right)^{\frac{r}{p}}}\right)^{\frac{1}{r}} \\
& \lesssim\left(\sum_{k \in \mathbb{Z}} \sup _{s \in\left[x_{k}, x_{k+1}\right]} \varphi(s)^{-\frac{r}{p}} W(s)^{\frac{r}{q}}\right)^{\frac{1}{r}} \\
\lesssim & \left(\sum_{k \in \mathbb{Z}} \frac{W\left(x_{k}\right)^{\frac{r}{q}}}{\varphi\left(x_{k}\right)^{\frac{r}{p}}}\right)^{\frac{1}{r}}+\left(\sum_{k \in \mathbb{Z}} \sup _{s \in\left[x_{k}, x_{k+1}\right]} \varphi(s)^{-\frac{r}{p}}\left(\int_{x_{k}}^{s} w(y) d y\right)^{\frac{r}{q}}\right)^{\frac{1}{r}} \\
\lesssim & \left(\sum_{k \in \mathbb{Z}} \frac{\left(\int_{x_{k}}^{x_{k+1}} w(s) d s\right)^{\frac{r}{q}}}{\varphi\left(x_{k}\right)^{\frac{r}{p}}}\right)^{\frac{1}{r}} \\
& +\left(\sum_{k \in \mathbb{Z}} \sup _{s \in\left[x_{k}, x_{k+1}\right]} \varphi(s)^{-\frac{r}{p}}\left(\int_{x_{k}}^{s} w(y) d y\right)^{\frac{r}{q}}\right)^{\frac{1}{r}}<\infty .
\end{aligned}
$$


Let $0<q<1$. For $k \in \mathbb{Z}$, define $h_{k}$ so that $\operatorname{supp} h_{k} \in\left[x_{k}, x_{k+1}\right]$, $\int_{x_{k}}^{x_{k+1}} h_{k}(s) \varphi(s)^{\frac{1}{p}} d s=1$, and

$$
\begin{aligned}
\int_{x_{k}}^{x_{k+1}}\left(\int_{t}^{x_{k+1}}\right. & \left.h_{k}(s) d s\right)^{q} w(t) d t \\
& \gtrsim\left(\int_{x_{k}}^{x_{k+1}}\left(\int_{x_{k}}^{t} w(s) d s\right)^{\frac{q}{1-q}} w(t) \varphi(t)^{-\frac{q}{p(1-q)}} d t\right)^{1-q} .
\end{aligned}
$$

This is possible thanks to the saturation of the Hardy inequality and due to a simple modification of [28, Theorem 3.3]. Let $\left\{a_{k}\right\}$ be a sequence of non-negative real numbers. We define

$$
h(s)=\sum_{k \in \mathbb{Z}} a_{k} h_{k}(s) .
$$

From (4.15) we get, using the definition of $t_{k}$ and $h$,

$$
\begin{array}{r}
\left(\sum_{k \in \mathbb{Z}} a_{k}^{q}\left(\int_{x_{k}}^{x_{k+1}}\left(\int_{x_{k}}^{t} w(s) d s\right)^{\frac{q}{1-q}} w(t) \varphi(t)^{-\frac{q}{p(1-q)}} d t\right)^{1-q}\right)^{\frac{1}{q}} \\
\lesssim\left(\sum_{k \in \mathbb{Z}} a_{k}^{p}\right)^{\frac{1}{p}}
\end{array}
$$

Let $p \leq q$. Then, by Proposition 4.1,

$$
\sup _{k \in \mathbb{Z}}\left(\int_{x_{k}}^{x_{k+1}}\left(\int_{x_{k}}^{t} w(s) d s\right)^{\frac{q}{1-q}} w(t) \varphi(t)^{-\frac{q}{p(1-q)}} d t\right)^{1-q}<\infty .
$$

By Lemma 3.8 and Lemma 3.3 we get

$$
\begin{aligned}
A(3) \lesssim & \sup _{k \in \mathbb{Z}}\left(\int_{x_{k}}^{x_{k+1}} W^{\frac{q}{1-q}}(t) w(t) \varphi(t)^{-\frac{q}{p(1-q)}} d t\right)^{\frac{1-q}{q}} \\
\lesssim & \sup _{k \in \mathbb{Z}} W\left(x_{k}\right)\left(\int_{x_{k}}^{x_{k+1}} w(t) \varphi(t)^{-\frac{q}{p(1-q)}} d t\right)^{\frac{1-q}{q}} \\
& +\sup _{k \in \mathbb{Z}}\left(\int_{x_{k}}^{x_{k+1}}\left(\int_{x_{k}}^{t} w(s) d s\right)^{\frac{q}{1-q}} w(t) \varphi(t)^{-\frac{q}{p(1-q)}} d t\right)^{\frac{1-q}{q}} .
\end{aligned}
$$


By (4.18), the second summand on the right hand side of (4.19) is finite. It remains to estimate the first term, that is, to show that also

$$
\sup _{k \in \mathbb{Z}} W\left(x_{k}\right)\left(\int_{x_{k}}^{x_{k+1}} w(t) \varphi(t)^{-\frac{q}{p(1-q)}} d t\right)^{\frac{1-q}{q}}<\infty .
$$

This is obvious when $\int_{x_{k}}^{x_{k+1}} w(s) d s \leq W\left(x_{k}\right)$. Assume that $\int_{x_{k}}^{x_{k+1}} w(s) d s>$ $W\left(x_{k}\right)$, and let $t_{k} \in\left[x_{k}, x_{k+1}\right]$ be such that $\int_{x_{k}}^{t_{k}} w(s) d s=W\left(x_{k}\right)$. Then, by (4.18)

$$
\begin{aligned}
& \sup _{k \in \mathbb{Z}} W\left(x_{k}\right)\left(\int_{x_{k}}^{x_{k+1}} w(t) \varphi(t)^{-\frac{q}{p(1-q)}} d t\right)^{\frac{1-q}{q}} \\
& \lesssim \sup _{k \in \mathbb{Z}} W\left(x_{k}\right)\left(\int_{x_{k}}^{t_{k}} w(t) \varphi(t)^{-\frac{q}{p(1-q)}} d t\right)^{\frac{1-q}{q}} \\
& +\sup _{k \in \mathbb{Z}} W\left(x_{k}\right)\left(\int_{t_{k}}^{x_{k+1}} w(t) \varphi(t)^{-\frac{q}{p(1-q)}} d t\right)^{\frac{1-q}{q}} \\
& \lesssim \sup _{k \in \mathbb{Z}} \varphi\left(x_{k}\right)^{-\frac{1}{p}} W\left(x_{k}\right)^{\frac{1}{q}} \\
& \quad+\sup _{k \in \mathbb{Z}}\left(\int_{t_{k}}^{x_{k+1}}\left(\int_{x_{k}}^{t} w(s) d s\right)^{\frac{q}{1-q}} w(t) \varphi(t)^{-\frac{q}{p(1-q)}} d t\right)^{\frac{1-q}{q}} .
\end{aligned}
$$

Now,

$$
\begin{aligned}
\sup _{k \in \mathbb{Z}} \varphi\left(x_{k}\right)^{-\frac{1}{p}} W\left(x_{k}\right)^{\frac{1}{q}} & \approx \sup _{k \in \mathbb{Z}} \varphi\left(x_{k}\right)^{-\frac{1}{p}}\left(\int_{x_{k-1}}^{x_{k}} w(s) d s\right)^{\frac{1}{q}} \\
& \leq \sup _{k \in \mathbb{Z}} \varphi\left(x_{k}\right)^{-\frac{1}{p}}\left(\int_{x_{k-1}}^{x_{k}}\left(\int_{x_{k-1}}^{t} w(s) d s\right)^{\frac{q}{1-q}} w(t) d t\right)^{\frac{1-q}{q}} \\
& \leq \sup _{k \in \mathbb{Z}}\left(\int_{x_{k-1}}^{x_{k}} \varphi(t)^{-\frac{q}{p(1-q)}}\left(\int_{x_{k-1}}^{t} w(s) d s\right)^{\frac{q}{1-q}} w(t) d t\right)^{\frac{1-q}{q}},
\end{aligned}
$$


and

$$
\begin{gathered}
\sup _{k \in \mathbb{Z}}\left(\int_{t_{k}}^{x_{k+1}}\left(\int_{x_{k}}^{t} w(s) d s\right)^{\frac{q}{1-q}} w(t) \varphi(t)^{-\frac{q}{p(1-q)}} d t\right)^{\frac{1-q}{q}} \\
\leq\left(\int_{x_{k}}^{x_{k+1}}\left(\int_{x_{k}}^{t} w(s) d s\right)^{\frac{q}{1-q}} w(t) \varphi(t)^{-\frac{q}{p(1-q)}} d t\right)^{\frac{1-q}{q}}<\infty
\end{gathered}
$$

Hence, $A(3)<\infty$.

Let now $q<p$, then we get, by (4.17) and Proposition 4.1,

$$
\left(\sum_{k \in \mathbb{Z}}\left(\int_{x_{k}}^{x_{k+1}}\left(\int_{x_{k}}^{t} w(s) d s\right)^{\frac{q}{1-q}} w(t) \varphi(t)^{-\frac{q}{p(1-q)}} d t\right)^{\frac{(1-q) r}{q}}\right)^{\frac{1}{r}}<\infty
$$

By Theorem 2.11 and Lemma 3.5, we get

$$
\begin{aligned}
A(4) \approx & \left(\sum_{k \in \mathbb{Z}} \frac{W\left(x_{k}\right)^{\frac{r}{q}}+U\left(x_{k}\right)^{r}\left(\int_{x_{k}}^{\infty} W(t)^{\frac{q}{1-q}} w(t) U(t)^{-\frac{q}{1-q}} d t\right)^{\frac{(1-q) r}{q}}}{\varphi\left(x_{k}\right)^{\frac{r}{p}}}\right)^{\frac{1}{r}} \\
\approx & \left.\left(\sum_{k \in \mathbb{Z}}\left(\int_{x_{k}}^{x_{k+1}} W(t)^{\frac{q}{1-q}} w(t) \varphi(t)^{-\frac{q}{p(1-q)}} d t\right)^{\frac{(1-q) r}{q}}\right)^{\frac{1}{r}}\right)^{\frac{1}{r}} \\
\approx & \left.\left.\left(\sum_{k \in \mathbb{Z}} W\left(x_{k}\right)^{r}\left(\int_{x_{k}}^{x_{k+1}} w(t) \varphi(t)^{-\frac{q}{p(1-q)}} d t\right)^{\frac{(1-q) r}{q}}\right)^{\frac{q}{1-q}} w(t) \varphi(t)^{-\frac{q}{p(1-q)}} d t\right)^{\frac{(1-q) r}{q}}\right)^{\frac{1}{r}} \\
& +\left(\sum _ { k \in \mathbb { Z } } \left(\int_{x_{k}}^{x_{k+1}}\left(\int_{x_{k}}^{t} w(s) d s\right)^{\frac{(1)}{2}}\right.\right.
\end{aligned}
$$

By (4.20), the second term is finite. 
Next, note that

$$
\begin{aligned}
& W\left(x_{k}\right)^{r}\left(\int_{x_{k}}^{x_{k+1}} w(t) \varphi(t)^{-\frac{q}{p(1-q)}} d t\right)^{\frac{(1-q) r}{q}} \\
\lesssim & \varphi\left(x_{k}\right)^{-\frac{r}{p}} W\left(x_{k}\right)^{\frac{r}{q}}+\left(\int_{x_{k}}^{x_{k+1}}\left(\int_{x_{k}}^{t} w(s) d s\right)^{\frac{q}{1-q}} w(t) \varphi(t)^{-\frac{q}{p(1-q)}} d t\right)^{\frac{(1-q) r}{q}} .
\end{aligned}
$$

Indeed, if $\int_{x_{k}}^{x_{k+1}} w(s) d s<W\left(x_{k}\right)$, then

$$
\begin{aligned}
& W\left(x_{k}\right)^{r}\left(\int_{x_{k}}^{x_{k+1}} w(t) \varphi(t)^{-\frac{q}{p(1-q)}} d t\right)^{\frac{(1-q) r}{q}} \\
& \quad \leq \varphi\left(x_{k}\right)^{-\frac{r}{p}} W\left(x_{k}\right)^{r}\left(\int_{x_{k}}^{x_{k+1}} w(t) d t\right)^{\frac{(1-q) r}{q}} \leq \varphi\left(x_{k}\right)^{-\frac{r}{p}} W\left(x_{k}\right)^{\frac{r}{q}} .
\end{aligned}
$$

Now assume that $\int_{x_{k}}^{x_{k+1}} w(s) d s \geq W\left(x_{k}\right)$. Let $t_{k} \in\left[x_{k}, x_{k+1}\right]$ be such that $\int_{x_{k}}^{t_{k}} w(s) d s=W\left(x_{k}\right)$. Then we have

$$
\begin{aligned}
W\left(x_{k}\right)^{r} & \left(\int_{x_{k}}^{x_{k+1}} w(t) \varphi(t)^{-\frac{q}{p(1-q)}} d t\right)^{\frac{(1-q) r}{q}} \\
\leq & W\left(x_{k}\right)^{r}\left(\int_{x_{k}}^{t_{k}} w(t) d t\right)^{\frac{(1-q) r}{q}} \varphi\left(x_{k}\right)^{-\frac{r}{p}} \\
& +\left(\int_{t_{k}}^{x_{k+1}}\left(\int_{x_{k}}^{t} w(s) d s\right)^{\frac{q}{1-q}} w(t) \varphi(t)^{-\frac{q}{p(1-q)}} d t\right)^{\frac{(1-q) r}{q}} \\
\lesssim & \varphi\left(x_{k}\right)^{-\frac{r}{p}} W\left(x_{k}\right)^{\frac{r}{q}} \\
& +\left(\int_{x_{k}}^{x_{k+1}}\left(\int_{x_{k}}^{t} w(s) d s\right)^{\frac{q}{1-q}} w(t) \varphi(t)^{-\frac{q}{p(1-q)}} d t\right)^{\frac{(1-q) r}{q}} .
\end{aligned}
$$


We thus have

(4.22)

$$
\begin{aligned}
& \left(\sum_{k \in \mathbb{Z}} W\left(x_{k}\right)^{r}\left(\int_{x_{k}}^{x_{k+1}} w(t) \varphi(t)^{-\frac{q}{p(1-q)}} d t\right)^{\frac{(1-q) r}{q}}\right)^{\frac{1}{r}} \\
& \leq\left(\sum_{k \in \mathbb{Z}} \varphi\left(x_{k}\right)^{-\frac{r}{p}} W\left(x_{k}\right)^{\frac{r}{q}}\right)^{\frac{1}{r}} \\
& \quad+\left(\sum_{k \in \mathbb{Z}}\left(\int_{x_{k}}^{x_{k+1}}\left(\int_{x_{k}}^{t} w(s) d s\right)^{\frac{q}{1-q}} w(t) \varphi(t)^{-\frac{q}{p(1-q)}} d t\right)^{\frac{(1-q) r}{q}}\right)^{\frac{1}{r}},
\end{aligned}
$$

and, using Lemma 3.1 (i),

$$
\begin{aligned}
& \left(\sum_{k \in \mathbb{Z}} W\left(x_{k}\right)^{\frac{r}{q}} \varphi\left(x_{k}\right)^{-\frac{r}{p}}\right)^{\frac{1}{r}} \\
& \approx\left(\sum_{k \in \mathbb{Z}}\left(\int_{x_{k}}^{x_{k+1}} w(s) d s\right)^{\frac{r}{q}} \varphi\left(x_{k+1}\right)^{-\frac{r}{p}}\right)^{\frac{1}{r}} \\
& \quad \lesssim\left(\sum_{k \in \mathbb{Z}}\left(\int_{x_{k}}^{x_{k+1}}\left(\int_{x_{k}}^{t} w(s) d s\right)^{\frac{q}{1-q}} w(t) \varphi(t)^{-\frac{q}{p(1-q)}} d t\right)^{\frac{(1-q) r}{q}}\right)^{\frac{1}{r}}
\end{aligned}
$$

which is finite by $(4.20)$.

Finally, combining (4.21), (4.23) and (4.22), we obtain $A(4)<\infty$.

The proof is complete.

\section{Embeddings of classical Lorentz spaces, type $\Gamma \hookrightarrow \Gamma$}

In this section we characterize the inequality

$$
\left(\int_{0}^{\infty} f_{u}^{* *}(t)^{q} w(t) d t\right)^{\frac{1}{q}} \leq C\left(\int_{0}^{\infty} f_{u}^{* *}(t)^{p} v(t) d t\right)^{\frac{1}{p}}
$$

where $p, q \in(0, \infty)$ and $u, v, w$ are weights. 
Our main result reads as follows.

Theorem 5.1. Let $p, q \in(0, \infty)$ and let $u, v, w$ be weights. Assume that $v(t) d t$ is a non-degenerate measure with respect to $U^{p}$.

(i) Let $0<p \leq q<\infty$. Then (5.1) holds if and only if

$$
A(6)=\sup _{t \in(0, \infty)} \frac{\left(W(t)+U(t)^{q} \int_{t}^{\infty} U(s)^{-q} w(s) d s\right)^{\frac{1}{q}}}{\left(V(t)+U(t)^{p} \int_{t}^{\infty} U(s)^{-p} v(s) d s\right)^{\frac{1}{p}}}<\infty
$$

(ii) Let $0<q<p<\infty$. Then (5.1) holds if and only if

$$
\begin{aligned}
& A(7)=\left(\int_{0}^{\infty} \frac{\left(W(t)+U(t)^{q} \int_{t}^{\infty} U(y)^{-q} w(y) d y\right)^{\frac{r}{q}}}{\left(V(t)+U(t)^{p} \int_{t}^{\infty} U(s)^{-p} v(s) d s\right)^{\frac{r}{p}+2}}\right. \\
&\left.\times V(t) \int_{t}^{\infty} U(s)^{-p} v(s) d s d\left(U^{p}\right)(t)\right)^{\frac{1}{r}}<\infty
\end{aligned}
$$

where, again, $r=\frac{p q}{p-q}$. Moreover, $A(7) \approx A(8)$, where

$$
A(8)=\left(\int_{0}^{\infty} \frac{\left(W(t)+U(t)^{q} \int_{t}^{\infty} U(s)^{-q} w(s) d s\right)^{\frac{r}{q}-1} w(t)}{\left(V(t)+U(t)^{p} \int_{t}^{\infty} U(s)^{-p} v(s) d s\right)^{\frac{r}{p}}} d t\right)^{\frac{1}{r}}<\infty .
$$

Proof: Sufficiency: First, in order to prove (5.1) it is enough to show

$$
\begin{aligned}
& \left(\int_{0}^{\infty}\left(\int_{0}^{\infty} \frac{U(s) h(s)}{U(s)+U(t)} d s\right)^{q} w(t) d t\right)^{\frac{1}{q}} \\
& \leq\left(\int_{0}^{\infty}\left(\int_{0}^{\infty} \frac{U(s) h(s)}{U(s)+U(t)} d s\right)^{p} v(t) d t\right)^{\frac{1}{p}} .
\end{aligned}
$$

Let $\varphi$ be defined as in (4.3) and let $\left\{x_{k}\right\}$ be a discretizing sequence for $\varphi$. Recall that then $\varphi\left(x_{k}\right) \uparrow \uparrow, \varphi\left(x_{k}\right) U^{-p}\left(x_{k}\right) \downarrow$, and there is a decomposition $\mathbb{Z}=\mathbb{Z}_{1} \cup \mathbb{Z}_{2}$ such that $\mathbb{Z}_{1} \cap \mathbb{Z}_{2}=\emptyset$ and for $k \in \mathbb{Z}_{1}$ and $t \in \Delta_{k}:=\left[x_{k}, x_{k+1}\right]$ we have $\varphi(t) \approx \varphi\left(x_{k}\right)$, while for $k \in \mathbb{Z}_{1}$ and $t \in \Delta_{k}$ we have $\varphi(t) U(t)^{-p} \approx$ 
$\varphi\left(x_{k}\right) U\left(x_{k}\right)^{-p}$. Вy (4.4),

$$
\begin{aligned}
& \int_{0}^{\infty}\left(\int_{0}^{\infty} \frac{U(s) h(s)}{U(s)+U(t)}\right)^{q} w(t) d t \\
& \lesssim \sum_{k \in \mathbb{Z}} \int_{x_{k}}^{x_{k+1}}\left(\int_{t}^{\infty} h(s) d s\right)^{q} w(t) d t \\
& +\int_{x_{k}}^{x_{k+1}}\left(\int_{0}^{t} h(s) U(s) d s\right)^{q} U(t)^{-q} w(t) d t \\
& \lesssim \sum_{k \in \mathbb{Z}_{1}} \int_{x_{k}}^{x_{k+1}} w(t) d t\left(\int_{x_{k}}^{\infty} h(s) d s\right)^{q} \\
& +\sum_{k \in \mathbb{Z}_{2}} \int_{x_{k}}^{x_{k+1}} w(t) d t\left(\int_{x_{k+1}}^{\infty} h(s) d s\right)^{q} \\
& +\sum_{k \in \mathbb{Z}_{2}} \int_{x_{k}}^{x_{k+1}}\left(\int_{t}^{x_{k+1}} h(s) d s\right)^{q} w(t) d t \\
& +\sum_{k \in \mathbb{Z}_{2}} \int_{x_{k}}^{x_{k+1}} U(t)^{-q} w(t) d t\left(\int_{0}^{x_{k+1}} U(s) h(s) d s\right)^{q} \\
& +\sum_{k \in \mathbb{Z}_{1}} \int_{x_{k}}^{x_{k+1}} U(t)^{-q} w(t) d t\left(\int_{0}^{x_{k}} U(s) h(s) d s\right)^{q} \\
& +\sum_{k \in \mathbb{Z}_{1}} \int_{x_{k}}^{x_{k+1}}\left(\int_{x_{k}}^{t} U(s) h(s) d s\right)^{q} U(t)^{-q} w(t) d t \\
& \approx \sum_{k \in \mathbb{Z}_{1}} \int_{x_{k}}^{x_{k+1}} w(t) d t\left(\int_{x_{k}}^{\infty} h(s) d s\right)^{q} \\
& +\sum_{k \in \mathbb{Z}_{1}} \int_{x_{k}}^{x_{k+1}} U(t)^{-q} w(t) d t\left(\int_{0}^{x_{k}} U(s) h(s) d s\right)^{q} \\
& +\sum_{k \in \mathbb{Z}_{2}} \int_{x_{k}}^{x_{k+1}} U(t)^{-q} w(t) d t\left(\int_{0}^{x_{k+1}} U(s) h(s) d s\right)^{q} \\
& +\sum_{k \in \mathbb{Z}_{2}} \int_{x_{k}}^{x_{k+1}} w(t) d t\left(\int_{x_{k+1}}^{\infty} h(s) d s\right)^{q} \\
& =\mathrm{I}+\mathrm{II}+\mathrm{III}+\mathrm{IV} \text {, }
\end{aligned}
$$

say. 
Let first $p \leq q$. Then

$$
\begin{aligned}
\mathrm{I}= & \sum_{k \in \mathbb{Z}_{1}} \int_{x_{k}}^{x_{k+1}} w(t) d t\left(\int_{x_{k}}^{\infty} h(s) d s\right)^{q} \\
\leq & \left(\sum_{k \in \mathbb{Z}_{1}}\left(\int_{x_{k}}^{\infty} h(s) d s\right)^{p} \varphi\left(x_{k}\right)\right)^{\frac{q}{p}} \sup _{k \in \mathbb{Z}_{1}} \varphi\left(x_{k}\right)^{-\frac{q}{p}} \int_{0}^{x_{k+1}} w(t) d t \\
\mathrm{II}= & \sum_{k \in \mathbb{Z}_{1}} \int_{x_{k}}^{x_{k+1}} U(t)^{-q} w(t) d t\left(\int_{0}^{x_{k}} U(s) h(s) d s\right)^{q} \\
\leq & \left(\sum_{k \in \mathbb{Z}_{1}}\left(\int_{0}^{x_{k}} U(s) h(s) d s\right)^{p} U\left(x_{k}\right)^{-p} \varphi\left(x_{k}\right)\right)^{\frac{q}{p}} \\
& \times \sup _{k \in \mathbb{Z}_{1}} \varphi\left(x_{k}\right)^{-\frac{q}{p}} U\left(x_{k}\right)^{q} \int_{x_{k}}^{\infty} w(t) U(t)^{-q} d t \\
\mathrm{III}= & \sum_{k \in \mathbb{Z}_{2}} \int_{x_{k}}^{x_{k+1}} U(t)^{-q} w(t) d t\left(\int_{0}^{x_{k+1}} U(s) h(s) d s\right)^{q} \\
\leq & \left(\sum_{k \in \mathbb{Z}_{2}}\left(\int_{0}^{x_{k+1}} U(s) h(s) d s\right)^{p} U\left(x_{k+1}\right)^{-p} \varphi\left(x_{k+1}\right)\right)^{\frac{q}{p}} \\
& \times \sup _{k \in \mathbb{Z}_{2}} \varphi\left(x_{k+1}\right)^{-\frac{q}{p}} U\left(x_{k+1}\right)^{q} \int_{x_{k}}^{\infty} w(t) U(t)^{-q} d t,
\end{aligned}
$$

and

$$
\begin{aligned}
\mathrm{IV}= & \sum_{k \in \mathbb{Z}_{2}} \int_{x_{k}}^{x_{k+1}} w(t) d t\left(\int_{x_{k+1}}^{\infty} h(s) d s\right)^{q} \\
\leq & \left(\sum_{k \in \mathbb{Z}_{2}}\left(\int_{x_{k+1}}^{\infty} h(s) d s\right)^{p} \varphi\left(x_{k+1}\right)\right)^{\frac{q}{p}} \\
& \times \sup _{k \in \mathbb{Z}_{2}} \varphi\left(x_{k+1}\right)^{-\frac{q}{p}} \int_{0}^{x_{k+1}} w(t) d t .
\end{aligned}
$$


Using the fact that

$$
\varphi\left(x_{k}\right) \approx \varphi\left(x_{k+1}\right) \quad \text { when } k \in \mathbb{Z}_{1}
$$

and

$$
\varphi\left(x_{k}\right) U\left(x_{k}\right)^{-p} \approx \varphi\left(x_{k+1}\right) U\left(x_{k+1}\right)^{-p} \quad \text { when } k \in \mathbb{Z}_{2},
$$

we get

$$
\begin{aligned}
\int_{0}^{\infty}\left(\int_{0}^{\infty} \frac{U(s)}{U(s)+U(t)} d s\right)^{q} w(t) d t & \\
& \lesssim A(6)^{q}\left(\int_{0}^{\infty}\left(\int_{0}^{\infty} \frac{U(s) h(s)}{U(s)+U(t)} d s\right)^{p} v(t) d t\right)^{q} .
\end{aligned}
$$

Now assume that $q<p$. Using the Hölder inequality with parameters $\frac{p}{q}$ and $\frac{p}{p-q}$, Lemma 3.1 (i), and (4.4), we obtain

$$
\begin{aligned}
\mathrm{I} \leq & \left(\sum_{k \in \mathbb{Z}_{1}}\left(\int_{x_{k}}^{\infty} h(s) d s\right)^{p} \varphi\left(x_{k}\right)\right)^{\frac{q}{p}} \\
& \times\left(\sum_{k \in \mathbb{Z}_{1}} W\left(x_{k+1}\right)^{\frac{r}{q}} \varphi\left(x_{k}\right)^{-\frac{r}{p}}\right)^{\frac{q}{r}} \\
\lesssim & \left(\int_{0}^{\infty}\left(\int_{0}^{\infty} \frac{U(s) h(s)}{U(s)+U(t)} d s\right)^{p} v(t) d t\right)^{\frac{q}{p}} \\
& \times\left(\sum_{k \in \mathbb{Z}_{1}} W\left(x_{k+1}\right)^{\frac{r}{q}} \varphi\left(x_{k+1}\right)^{-\frac{r}{p}}\right)^{\frac{q}{r}} \\
\lesssim & \left(\int_{0}^{\infty}\left(\int_{0}^{\infty} \frac{U(s) h(s)}{U(s)+U(t)} d s\right)^{p} v(t) d t\right)^{\frac{q}{p}} \\
& \times\left(\sum_{k \in \mathbb{Z}} W\left(x_{k}\right)^{\frac{r}{q}} \varphi\left(x_{k}\right)^{-\frac{r}{p}}\right)^{\frac{q}{r}},
\end{aligned}
$$




$$
\begin{aligned}
\mathrm{II} \leq & \left(\sum_{k \in \mathbb{Z}_{1}}\left(\int_{0}^{x_{k}} U(s) h(s) d s\right)^{p} U\left(x_{k}\right)^{-p} \varphi\left(x_{k}\right)\right)^{\frac{q}{p}} \\
& \times\left(\sum_{k \in \mathbb{Z}_{1}}\left(\int_{x_{k}}^{\infty} w(t) U(t)^{-q} d t\right)^{\frac{r}{q}} \varphi\left(x_{k}\right)^{-\frac{r}{p}} U\left(x_{k}\right)^{r}\right)^{\frac{q}{r}} \\
\leq & \left(\int_{0}^{\infty}\left(\int_{0}^{\infty} \frac{U(s) h(s)}{U(s)+U(t)} d s\right)^{p} v(t) d t\right)^{\frac{q}{p}} \\
& \times\left(\sum_{k \in \mathbb{Z}_{1}}\left(\int_{x_{k}}^{\infty} w(t) U(t)^{-q} d t\right)^{\frac{r}{q}} \varphi\left(x_{k}\right)^{-\frac{r}{p}} U\left(x_{k}\right)^{r}\right)^{\frac{q}{r}} \\
\mathrm{III} \leq & \left(\sum_{k \in \mathbb{Z}_{2}}\left(\int_{0}^{x_{k+1}} U(s) h(s) d s\right)^{p} U\left(x_{k+1}\right)^{-p} \varphi\left(x_{k+1}\right)\right)^{\frac{q}{p}} \\
& \times\left(\sum_{k \in \mathbb{Z}_{2}}\left(\int_{x_{k}}^{\infty} w(t) U(t)^{-q} d t\right)^{\frac{r}{q}} \varphi\left(x_{k+1}\right)^{-\frac{r}{p}} U\left(x_{k+1}\right)^{r}\right)^{\frac{r}{q}} \\
\lesssim & \left(\int_{0}^{\infty}\left(\int_{0}^{\infty} \frac{U(s) h(s)}{U(s)+U(t)} d s\right)^{p} v(t) d t\right)^{\frac{q}{p}} \\
& \times\left(\sum_{k \in \mathbb{Z}_{2}}\left(\int_{x_{k}}^{\infty} w(t) U(t)^{-q} d t\right)^{\frac{r}{q}} \varphi\left(x_{k}\right)^{-\frac{r}{p}} U\left(x_{k}\right)^{r}\right)^{\frac{q}{r}}
\end{aligned}
$$

and

$$
\begin{aligned}
\mathrm{IV} \leq & \left(\sum_{k \in \mathbb{Z}_{2}}\left(\int_{x_{k+1}}^{\infty} h(s) d s\right)^{p} \varphi\left(x_{k+1}\right)\right)^{\frac{q}{p}} \\
& \times\left(\sum_{k \in \mathbb{Z}_{2}}\left(\int_{0}^{x_{k+1}} w(t) d t\right)^{\frac{r}{q}} \varphi\left(x_{k+1}\right)^{-\frac{r}{p}}\right)^{\frac{q}{r}} \\
\leq & \left(\int_{0}^{\infty}\left(\int_{0}^{\infty} \frac{U(s) h(s)}{U(s)+U(t)} d s\right)^{p} v(t) d t\right)^{\frac{q}{p}} \\
& \times\left(\sum_{k \in \mathbb{Z}}\left(\int_{0}^{x_{k}} w(t) d t\right)^{\frac{r}{q}} \varphi\left(x_{k}\right)^{-\frac{r}{p}}\right)^{\frac{q}{r}} .
\end{aligned}
$$


Combining all these estimates with Theorem 2.15, we get the desired estimate

$$
\begin{gathered}
\left(\int_{0}^{\infty}\left(\int_{0}^{\infty} \frac{U(s) h(s)}{U(s)+U(t)} d s\right)^{q} w(t) d t\right)^{\frac{1}{q}} \\
\leq A(7)\left(\int_{0}^{\infty}\left(\int_{0}^{\infty} \frac{U(s) h(s)}{U(s)+U(t)} d s\right)^{p} v(t) d t\right)^{\frac{1}{p}}
\end{gathered}
$$

Necessity: We still assume that $\left\{x_{k}\right\}$ is a discretizing sequence for the function $\varphi$ from (4.3). From (5.2) we have

$$
\begin{aligned}
& \left(\sum_{k \in \mathbb{Z}_{1}} \int_{x_{k}}^{x_{k+1}}\left(\int_{t}^{x_{k+1}} h(s) d s\right)^{q} w(t) d t\right. \\
& \left.\quad+\sum_{k \in \mathbb{Z}_{2}} \int_{x_{k}}^{x_{k+1}}\left(\int_{x_{k}}^{t} h(s) U(s) d s\right)^{q} U(t)^{-q} w(t) d t\right)^{\frac{1}{q}} \\
& \leq\left(\sum_{k \in \mathbb{Z}_{1}}\left(\int_{x_{k}}^{x_{k+1}} h(s) d s\right)^{p} \varphi\left(x_{k}\right)\right. \\
& \left.\quad+\sum_{k \in \mathbb{Z}_{2}}\left(\int_{x_{k}}^{x_{k+1}} h(s) U(s) d s\right)^{p} U\left(x_{k}\right)^{-p} \varphi\left(x_{k}\right)\right)^{\frac{1}{p}}
\end{aligned}
$$

Let $t_{k} \in\left[x_{k}, x_{k+1}\right]$ be such that, for $k \in \mathbb{Z}_{1}$,

$$
\int_{x_{k}}^{t_{k}} w(s) d s=\frac{1}{2} \int_{x_{k}}^{x_{k+1}} w(s) d s
$$

and, for $k \in \mathbb{Z}_{2}$,

$$
\int_{x_{k}}^{t_{k}} U(s)^{-q} w(s) d s=\frac{1}{2} \int_{x_{k}}^{x_{k+1}} U(s)^{-q} w(s) d s .
$$

Let $\left\{a_{k}\right\}$ be a sequence of non-negative real numbers. We define

$$
h(x)=\sum_{k \in \mathbb{Z}_{1}} \frac{a_{k}}{x_{k+1}-t_{k}} \chi_{\left[t_{k}, x_{k+1}\right]}(x)+\sum_{k \in \mathbb{Z}_{2}} \frac{a_{k}}{\int_{x_{k}}^{t_{k}} U(s) d s} \chi_{\left[x_{k}, t_{k}\right]}(x) .
$$


Now, we get from (5.3)

$$
\begin{aligned}
\left(\sum_{k \in \mathbb{Z}_{1}} a_{k}^{q} \int_{x_{k}}^{x_{k+1}} w(t) d t\right. & \left.+\sum_{k \in \mathbb{Z}_{2}} a_{k}^{q} \int_{x_{k}}^{x_{k+1}} U(t)^{-q} w(t) d t\right)^{\frac{1}{q}} \\
& \lesssim\left(\sum_{k \in \mathbb{Z}_{1}} a_{k}^{q} \varphi\left(x_{k}\right)+\sum_{k \in \mathbb{Z}_{2}} a_{k}^{p} U\left(x_{k}\right)^{-p} \varphi\left(x_{k}\right)\right)^{\frac{1}{p}}
\end{aligned}
$$

for every sequence $\left\{a_{k}\right\}$.

Assume first that $p \leq q$. Then, by Lemma 3.8 and Proposition 4.1,

$$
\begin{aligned}
A(6)<\sup _{k \in \mathbb{Z}_{1}} & \left(\int_{x_{k}}^{x_{k+1}} w(t) d t\right)^{\frac{1}{q}} \varphi\left(x_{k}\right)^{-\frac{1}{p}} \\
& +\sup _{k \in \mathbb{Z}_{2}}\left(\int_{x_{k}}^{x_{k+1}} U(t)^{-q} w(t) d t\right)^{\frac{1}{q}} U\left(x_{k}\right)^{r} \varphi\left(x_{k}\right)^{-\frac{1}{p}}<\infty .
\end{aligned}
$$

Now, let $q<p$. Then

$$
\begin{aligned}
\left(\sum_{k \in \mathbb{Z}_{1}}\left(\int_{x_{k}}^{x_{k+1}} w(t) d t\right)^{\frac{r}{q}} \varphi\left(x_{k}\right)^{-\frac{r}{p}}\right. \\
\left.\quad+\sum_{k \in \mathbb{Z}_{2}}\left(\int_{x_{k}}^{x_{k+1}} U(t)^{-q} w(t) d t\right)^{\frac{r}{q}} U\left(x_{k}\right)^{r} \varphi\left(x_{k}\right)^{-\frac{r}{p}}\right)^{\frac{1}{r}}<\infty .
\end{aligned}
$$

By Theorem 2.15 and Proposition 4.1,

$$
\begin{aligned}
A(7) \approx & \left(\sum_{k \in \mathbb{Z}}\left(W\left(x_{k}\right)+U\left(x_{k}\right)^{q} \int_{x_{k}}^{\infty} U(t)^{-q} w(t) d t\right)^{\frac{r}{q}} \varphi\left(x_{k}\right)^{-\frac{r}{p}}\right)^{\frac{1}{r}} \\
\approx & \left(\sum_{k \in \mathbb{Z}_{1}}\left(\int_{x_{k}}^{x_{k+1}} w(t) d t\right)^{\frac{r}{q}} \varphi\left(x_{k}\right)^{-\frac{r}{p}}\right. \\
& \left.+\sum_{k \in \mathbb{Z}_{2}}\left(\int_{x_{k}}^{x_{k+1}} U(t)^{-q} w(t) d t\right)^{\frac{r}{q}} \varphi\left(x_{k}\right)^{-\frac{r}{p}} U\left(x_{k}\right)^{r}\right)^{\frac{1}{r}},
\end{aligned}
$$

hence $A(7)<\infty$. Now, $A(7) \approx A(8)$ by Theorem 2.11 , Theorem 2.15 and Lemma 3.5. The proof is complete. 


\section{Associate spaces of the spaces $\Gamma^{p}(v)$}

Here we present a characterization of the associate space of

$$
\Gamma_{u}^{p}(v)=\left\{f \in \mathcal{M}(\mathcal{R}, \mu) ;\|f\|_{\Gamma_{u}^{p}(v)}:=\left(\int_{0}^{\infty}\left(f_{u}^{* *}(t)\right)^{p} v(t) d t\right)^{1 / p}<\infty\right\},
$$

where $u, v$ are weights and $p \in(0, \infty)$.

Theorem 6.1. Let $u, v$ be positive weights on $[0, \infty)$. Let $p \in(0, \infty)$. Set $X=\Gamma_{u}^{p}(v)$ and let $X^{\prime}$ denote its associate space, that is,

$$
\|g\|_{X^{\prime}}=\sup \left\{\int_{0}^{\infty} f^{*}(t) g^{*}(t) d t ;\|f\|_{X} \leq 1\right\} .
$$

(i) Let $0<p \leq 1$. Then

$$
\|g\|_{X^{\prime}} \approx \sup _{t \in(0, \infty)} \frac{\int_{0}^{t} g^{*}(s) d s}{\left(V(t)+U(t)^{p} \int_{t}^{\infty} U(s)^{-p} v(s) d s\right)^{\frac{1}{p}}} .
$$

(ii) Let $1<p<\infty$. Then

$$
\begin{gathered}
\|g\|_{X^{\prime}} \approx\left(\int_{0}^{\infty} \frac{\left[\sup _{y \in[t, \infty)} U(y)^{-p^{\prime}}\left(\int_{0}^{y} g^{*}(\tau) d \tau\right)^{p^{\prime}}\right]}{\left(V(t)+U(t)^{p} \int_{t}^{\infty} U(s)^{-p} v(s) d s\right)^{p^{\prime}+1}}\right. \\
\left.\times U(t)^{p^{\prime}} V(t) \int_{t}^{\infty} U(s)^{-p} v(s) d s d\left(U^{p}\right)(t)\right)^{\frac{1}{p^{\prime}}} .
\end{gathered}
$$

Proof: This is just a simple application of Theorem 4.2 to $q=1$ and $w=g^{*}$.

In the classical situation (that is, when $u \equiv 1$ ), the associate norm can be characterized by a little simpler expression, which is worth pointing out.

Theorem 6.2. Let $v$ be a positive weight on $[0, \infty)$. Let $p \in(0, \infty)$. Denote $X=\Gamma^{p}(v)$ and $X^{\prime}$ its associate space.

(i) Let $0<p \leq 1$. Then

$$
\|g\|_{X^{\prime}} \approx \sup _{t \in(0, \infty)} \frac{\int_{0}^{t} g^{*}(s) d s}{\left(V(t)+t^{p} \int_{t}^{\infty} s^{-p} v(s) d s\right)^{\frac{1}{p}}}
$$


(ii) Let $1<p<\infty$. Then

$$
\|g\|_{X^{\prime}} \approx\left(\int_{0}^{\infty} g^{* *}(t)^{p^{\prime}} \frac{t^{p^{\prime}+p-1} V(t) \int_{t}^{\infty} s^{-p} v(s) d s}{\left(V(t)+t^{p} \int_{t}^{\infty} s^{-p} v(s) d s\right)^{p^{\prime}+1}} d t\right)^{\frac{1}{p^{\prime}}}
$$

Remark 6.3. Let us recall that the following special case of Theorem 6.2 was proved in [12, Theorem 2.7]:

Assume that

$$
V(t) \lesssim t^{p} \int_{t}^{\infty} s^{-p} v(s) d s, \quad t \in(0, \infty) .
$$

(This is satisfied for example when $v$ is non-decreasing on $(0, \infty)$.) Then

$$
\|g\|_{\left(\Gamma^{p}(v)\right)^{\prime}} \approx\left(\int_{0}^{\infty} \frac{g^{* *}(t)^{p^{\prime}}}{\left(\int_{t}^{\infty} s^{-p} v(s) d s\right)^{p^{\prime}-1}} \frac{d t}{t}\right)^{\frac{1}{p^{\prime}}} .
$$

From Theorem 6.2 we can however also obtain a converse assertion: Assume that

$$
V(t) \gtrsim t^{p} \int_{t}^{\infty} s^{-p} v(s) d s, \quad t \in(0, \infty) .
$$

(This is satisfied for example when $v$ is non-increasing on $(0, \infty)$ and moreover $v(t) t \lesssim V(t)$ for $t \in(0, \infty)$.) Then

$$
\|g\|_{\left(\Gamma^{p}(v)\right)^{\prime}} \approx\left(\int_{0}^{\infty} t^{p^{\prime}-1} \frac{g^{* *}(t)^{p^{\prime}}}{V(t)^{p^{\prime}-1}}\right)^{\frac{1}{p^{\prime}}} .
$$

Remark 6.4. When this paper was already written, it was brought to our attention that G. Sinnamon has recently obtained another characterization of the dual of $\Gamma^{p}(v)$.

\section{References}

[1] M. A. Ariño And B. Muckenhoupt, Maximal functions on classical Lorentz spaces and Hardy's inequality with weights for nonincreasing functions, Trans. Amer. Math. Soc. 320(2) (1990), $727-735$.

[2] S. Barza, L.-E. Persson and J. Soria, Sharp weighted multidimensional integral inequalities for monotone functions, Math. Nachr. 210 (2000), 43-58.

[3] C. Bennett and R. Sharpley, "Interpolation of operators", Pure and Applied Mathematics 129, Academic Press, Inc., Boston, MA, 1988. 
[4] J. Bergh, V. Burenkov And L.-E. Persson, Best constants in reversed Hardy's inequalities for quasimonotone functions, Acta Sci. Math. (Szeged) 59(1-2) (1994), 221-239.

[5] J. Bergh AND J. LÖFströM, "Interpolation spaces. An introduction", Grundlehren der Mathematischen Wissenschaften 223, Springer-Verlag, Berlin-New York, 1976.

[6] M. J. Carro, A. García del Amo And J. Soria, Weak-type weights and normable Lorentz spaces, Proc. Amer. Math. Soc. 124(3) (1996), 849-857.

[7] M. J. Carro, L. Pick, J. Soria and V. D. Stepanov, On embeddings between classical Lorentz spaces, Math. Inequal. Appl. 4(3) (2001), 397-428.

[8] M. J. Carro And J. Soria, Weighted Lorentz spaces and the Hardy operator, J. Funct. Anal. 112(2) (1993), 480-494.

[9] M. J. CARro And J. Soria, Boundedness of some integral operators, Canad. J. Math. 45(6) (1993), 1155-1166.

[10] M. J. Carro And J. Soria, The Hardy-Littlewood maximal function and weighted Lorentz spaces, J. London Math. Soc. (2) 55(1) (1997), 146-158.

[11] A. Cianchi, R. Kerman, B. Opic And L. Pick, A sharp rearrangement inequality for the fractional maximal operator, Studia Math. 138(3) (2000), 277-284.

[12] D. E. Edmunds, R. Kerman And L. Pick, Optimal Sobolev imbeddings involving rearrangement-invariant quasinorms, $J$. Funct. Anal. 170(2) (2000), 307-355.

[13] A. Gogatishvili, B. Opic And L. Pick, Weighted inequalities for Hardy-type operators involving suprema, Charles University, Faculty of Mathematics and Physics, Preprint No. MATH-KMA-2001/54, Prague (2001), 1-24, URL address: http://www.karlin.mff.cuni.cz/ ${ }^{\sim}$ rokyta/preprint/index.php.

[14] M. L. GOL'DMAN, Integral inequalities on a cone of functions with monotonicity properties, (Russian), Dokl. Akad. Nauk SSSR 320(5) (1991), 1037-1042; translation in Soviet Math. Dokl. 44(2) (1992), $581-587$.

[15] M. L. Gol'dman, On integral inequalities on the set of functions with some properties of monotonicity, in: "Function spaces, differential operators and nonlinear analysis" (Friedrichroda, 1992), Teubner-Texte Math. 133, Teubner, Stuttgart, 1993, pp. 274-279.

[16] M. L. Gol'dman, H. P. Heinig and V. D. Stepanov, On the principle of duality in Lorentz spaces, Canad. J. Math. 48(5) (1996), 959-979. 
[17] K.-G. Grosse-Erdmann, "The blocking technique, weighted mean operators and Hardy's inequality", Lecture Notes in Mathematics 1679, Springer-Verlag, Berlin, 1998.

[18] H. P. Heinig and L. Maligranda, Weighted inequalities for monotone and concave functions, Studia Math. 116(2) (1995), $133-165$.

[19] R. Kerman and L. Pick, Optimal Sobolev imbedding spaces, Charles University, Faculty of Mathematics and Physics, Preprint No. MATH-KMA-2002/93, Prague (2002), 1-44, URL address: http://www.karlin.mff.cuni.cz/ rokyta/preprint/index.php.

[20] S. LAI, Weighted norm inequalities for general operators on monotone functions, Trans. Amer. Math. Soc. 340(2) (1993), 811-836.

[21] G. G. Lonentz, On the theory of spaces $\Lambda$, Pacific J. Math. 1 (1951), 411-429.

[22] L. Maligranda, Weighted inequalities for quasi-monotone functions, J. London Math. Soc. (2) 57(2) (1998), 363-370.

[23] C. J. Neugebauer, Weighted norm inequalities for averaging operators of monotone functions, Publ. Mat. 35(2) (1991), 429-447.

[24] B. Opic And A. Kufner, "Hardy-type inequalities", Pitman Research Notes in Mathematics Series 219, Longman Scientific \& Technical, Harlow, 1990.

[25] K. I. Oskolkov, Approximation properties of integrable functions on sets of full measure, (Russian), Mat. Sb. (N.S.) 103(145) (1977), no. $4,563-589,631$.

[26] E. SAWyER, Boundedness of classical operators on classical Lorentz spaces, Studia Math. 96(2) (1990), 145-158.

[27] G. Sinnamon, Spaces defined by the level function and their duals, Studia Math. 111(1) (1994), 19-52.

[28] G. Sinnamon and V. D. Stepanov, The weighted Hardy inequality: new proofs and the case $p=1, J$. London Math. Soc. (2) 54(1) (1996), 89-101.

[29] J. Soria, Lorentz spaces of weak-type, Quart. J. Math. Oxford Ser. (2) 49(193) (1998), 93-103.

[30] V. D. Stepanov, The weighted Hardy's inequality for nonincreasing functions, Trans. Amer. Math. Soc. 338(1) (1993), 173-186.

[31] V. D. Stepanov, Integral operators on the cone of monotone functions, J. London Math. Soc. (2) 48(3) (1993), 465-487. 
[32] V. D. Stepanov, Integral operators on the cone of monotone functions and embeddings of Lorentz spaces, (Russian), Dokl. Akad. Nauk SSSR 317(6) (1991), 1308-1311; translation in Soviet Math. Dokl. 43(2) (1991), 620-623.

Amiran Gogatishvili:

Mathematical Institute

Czech Academy of Sciences

Žitná 25

11567 Praha 1

Czech Republic

E-mail address: gogatish@math.cas.cz

Luboš Pick:

Department of Mathematical Analysis

Faculty of Mathematics and Physics

Charles University

Sokolovská 83

18675 Praha 8

Czech Republic

E-mail address: pick@karlin.mff.cuni.cz

Primera versió rebuda el 18 d'abril de 2002, darrera versió rebuda el 10 d'octubre de 2002. 